Homology, Homotopy and Applications, vol.18(2), 2016, pp.1-29

\title{
THE HOMOLOGY OF tmf
}

\author{
AKHIL MATHEW
}

(communicated by J.P.C. Greenlees)

\begin{abstract}
We compute the mod 2 homology of the spectrum tmf of topological modular forms by proving a 2-local equivalence $\operatorname{tmf} \wedge D A(1) \simeq \operatorname{tmf}_{1}(3) \simeq B P\langle 2\rangle$, where $D A(1)$ is an eight cell complex whose cohomology doubles the subalgebra $\mathcal{A}(1)$ of the Steenrod algebra generated by $\mathrm{Sq}^{1}$ and $\mathrm{Sq}^{2}$. To do so, we give, using the language of stacks, a modular description of the elliptic homology of $D A(1)$ via level three structures. We briefly discuss analogs at odd primes and recover the stack-theoretic description of the Adams-Novikov spectral sequence for tmf.
\end{abstract}

\section{Introduction}

\subsection{Results}

Let tmf be the $E_{\infty}$-ring spectrum of connective topological modular forms of Goerss, Hopkins, Mahowald, Miller, and Lurie. See [10] for a survey and [29, 7] for detailed treatments. The spectrum tmf is constructed from a derived version of the moduli stack $M_{\overline{e l l}}$ of elliptic curves, and its homotopy groups approximate both the stable homotopy groups of spheres and the ring of integral modular forms.

The primary goal of this paper is to compute the mod 2 cohomology of tmf, as a module over the Steenrod algebra. Namely, we give a proof of the following result:

Theorem 1.1 (Hopkins-Mahowald [14]). There is an isomorphism

$$
H^{*}(\operatorname{tmf} ; \mathbb{Z} / 2) \simeq \mathcal{A} / / \mathcal{A}(2) \stackrel{\text { def }}{=} \mathcal{A} \otimes_{\mathcal{A}(2)} \mathbb{Z} / 2,
$$

where $\mathcal{A}$ is the mod 2 Steenrod algebra and $\mathcal{A}(2) \subset \mathcal{A}$ is the subalgebra generated by $\mathrm{Sq}^{1}, \mathrm{Sq}^{2}, \mathrm{Sq}^{4}$.

The computation is carried out by exhibiting a 2-local eight cell complex $D A(1)$ and demonstrating an equivalence (also due to [14]),

$$
\operatorname{tmf} \wedge D A(1) \simeq B P\langle 2\rangle,
$$

which is a tmf-analog of Wood's theorem $k o \wedge \Sigma^{-2} \mathbb{C P}^{2} \simeq k u$. This equivalence implies the result on $H^{*}(\operatorname{tmf} ; \mathbb{Z} / 2)$ using Hopf algebra manipulations, and it enables the description of the Adams-Novikov spectral sequence of tmf via the Weierstrass Hopf algebroid or the moduli stack of cubic curves.

Received December 8, 2015; published on June 15, 2016.

2010 Mathematics Subject Classification: 55P42, 55P43.

Key words and phrases: topological modular form, algebraic stack, Steenrod algebra.

Article available at http://dx.doi.org/10.4310/HHA.2016.v18.n2.a1

Copyright (C) 2016, International Press. Permission to copy for private use granted. 
The form of $B P\langle 2\rangle$ encountered can be identified with the spectrum $\operatorname{tmf}_{1}(3)$ of connective topological modular forms of level 3 studied by Hill-Lawson [12] and by Lawson-Naumann [18]. In particular, we prove:

Theorem 1.2. At the prime 2 , we have an equivalence of tmf-modules

$$
\operatorname{tmf} \wedge D A(1) \simeq \operatorname{tmf}_{1}(3)
$$

At the prime 3 , there is an analog as well: one has a three cell complex $X_{3}$ such that

$$
\operatorname{tmf} \wedge X_{3} \simeq \operatorname{tmf}_{1}(2)
$$

\subsection{Methods}

We now give a brief overview of the method of proof used in this paper.

Suppose we knew the homology of tmf, i.e., (1). In this case, one could construct the eight cell complex $D A(1)$ such that, as an $\mathcal{A}(2)$-module, we have

$$
H^{*}(D A(1) ; \mathbb{Z} / 2) \simeq \mathcal{A}(2) /\left(\mathrm{Sq}^{1}\right) .
$$

We would then see by direct computation that tmf $\wedge D A(1)$ had the same homology as $B P\langle 2\rangle$ and could produce the equivalence (2) with some more effort (or by appealing to [3] at least after 2-completion). In this paper, we will work in reverse: we will prove (2) directly by working with stacks and then deduce the description of the homology.

Our proof of (2) proceeds by first replacing tmf by Tmf, the non-connective, nonperiodic version of tmf, and by working with the derived version of $M_{\overline{e l l}}$. In particular, we calculate $\operatorname{Tmf}_{*}(D A(1))$. To do so, we describe the module $E_{0}(D A(1))$, for $E$ an elliptic homology theory, in terms of $M_{\overline{\text { ell }}}$ : in other words, we identify a certain vector bundle on the moduli stack of elliptic curves as arising from an eight-fold cover of $\left(M_{\overline{\text { ell }}}\right)_{(2)}$.

Technical theorem. Let $E$ be an elliptic homology theory associated to a generalized elliptic curve $C \rightarrow \operatorname{Spec}(R)$, classified by a flat map $\operatorname{Spec}(R) \rightarrow M_{\overline{\text { ell }}}$. Then there is a natural identification of $R=E_{0}$-modules between $E_{0}(D A(1))$ and the universal $R$ algebra over which $C$ acquires a $\Gamma_{1}(3)$-structure.

This proof of identification relies on a basic technical trick, of which we explain a more elementary form.

Construction. Consider the scheme $\mathbb{P}_{\mathbb{C}}^{n}=\left(\operatorname{Spec}\left(\mathbb{C}\left[x_{0}, x_{1}, \ldots, x_{n}\right]\right) \backslash(0, \ldots, 0)\right) / \mathbb{G}_{m}$. This scheme is very well-behaved; it is smooth and proper. It is an open substack of the Artin stack $\mathfrak{X} \subset \operatorname{Spec}\left(\mathbb{C}\left[x_{0}, x_{1}, \ldots, x_{n}\right]\right) / \mathbb{G}_{m}$.

On the one hand, the geometry of $\mathfrak{X}$ is much worse than that of $\mathbb{P}_{\mathbb{C}}^{n}$ : it has a special point $x$, given by the image of the origin, whose stabilizer is a $\mathbb{G}_{m}$. On the other hand, the study of vector bundles or coherent sheaves on $\mathfrak{X}$ is vastly simpler than the analogous study on $\mathbb{P}_{\mathbb{C}}^{n}$, as they are given by graded modules over a polynomial ring. As an example, one has an analog of Nakayama's lemma:

Lemma. Any morphism of coherent sheaves $\mathcal{F} \rightarrow \mathcal{G}$ on $\mathfrak{X}$ inducing a surjection at the point $x$ is a surjection globally. 
In this paper, we will work with the moduli stack $M_{\overline{e l l}}$ of generalized elliptic curves, which is analogous to $\mathbb{P}_{\mathbb{C}}^{n}$ : it has good geometry, but it generally hard to study vector bundles on it. There is, however, another moduli stack $M_{c u b}$ classifying cubic curves, which contains $M_{\overline{e l l}}$ as an open substack. As in the above analogy, $M_{c u b}$ has a distinguished point with a bigger stabilizer for which one can prove an analog of Nakayama's lemma. Our main identification relies on the observation that the association $E \mapsto E_{0}(D A(1))$, which defines a vector bundle on $\left(M_{\overline{e l l}}\right)_{(2)}$, actually canonically extends to the larger stack $\left(M_{c u b}\right)_{(2)}$. As a result, it is possible to get a handle on this vector bundle using the distinguished point in $M_{c u b}$. After identifying the vector bundle, we use the descent spectral sequence to compute $\operatorname{Tmf}_{*}(D A(1))$. Finally, the description of $\operatorname{tmf}_{*}(D A(1))$ from that of $\operatorname{Tmf}_{*}(D A(1))$ follows from the gap theorem in $\pi_{*}$ Tmf.

The above summarizes the key technical work in the paper. In the rest of the paper, we calculate the homology $H_{*}(\operatorname{tmf} ; \mathbb{Z} / 2)$ by studying the map tmf $\rightarrow \operatorname{tmf} \wedge D A(1) \simeq$ $B P\langle 2\rangle$ and determining the image in homology, as the homology of $B P\langle 2\rangle$ is known as a subalgebra of the dual Steenrod algebra. The precise determination of the image relies on a few techniques with Hopf algebras.

This paper is organized as follows. Section 2 reviews the language of stacks and, in particular, the role of the moduli stack $M_{F G}$ of formal groups and states the results we need about tmf. Section 3 is purely algebraic and describes a vector bundle on the stack $M_{\overline{e l l}}$. Section 4 shows that this vector bundle arises from the eight cell complex $D A(1)$ and discusses the analog at odd primes. Section 5 contains the main remaining computations, in particular, of the homology.

\subsection{Previous work}

There is a significant literature on tmf and the homology of tmf has certainly been treated before. For instance, the notes of Rezk [29, sec. 20-21] give an entirely different approach to the calculation of $H_{*}(\mathrm{tmf} ; \mathbb{Z} / 2)$. Rezk's starting point is different from ours; we take the description via sheaves of spectra as given, whereas Rezk assumes the tmf-homology of the Thom spectrum $X(4)$. In particular, the calculation of $M U_{*}$ tmf is carried out in [29, Prop. 20.4] and we will reprove it here as well.

The equivalence (2) is due to Hopkins-Mahowald. The existence of an eight cell complex $D A(1)$ with the property that tmf $\wedge D A(1)$ is complex-orientable arises from the heuristic that the moduli stack of cubic curves has an eight-fold cover at the prime 2 which is the quotient by $\mathbb{G}_{m}$ of an affine scheme (cf. the notes to Hopkins's talk in [7, Ch. 9]).

\section{Acknowledgments}

I would like to thank heartily Mike Hopkins for suggesting this project and sharing his ideas. I would also like to thank Mark Behrens, Dustin Clausen, Tyler Lawson, Jacob Lurie, Lennart Meier, Niko Naumann, and Vesna Stojanoska for helpful discussions, and the referee for numerous comments. 


\section{The language of stacks}

Let $X$ be a spectrum. The homotopy groups $\pi_{*} X$ may be complicated, but often their calculation can be attacked by choosing an appropriate resolution of $X$ by simpler spectra. This formalism can be expressed efficiently using the language of stacks. The language has been described in the course notes $[13,20]$ and in the talk of Hopkins [7, Ch. 7]. Other references on the this viewpoint, especially on the moduli stack of formal groups, are [27] and [9]. We will briefly summarize what we need below.

\subsection{Stacks and spectra}

Suppose that $R$ is a fixed $A_{\infty}$-ring spectrum. The structure on $R$ enables one to build the cobar construction, a cosimplicial spectrum $\mathrm{CB}^{\bullet}(R)$ with $\mathrm{CB}^{s}(R)=$ $R^{\wedge(s+1)}$ and with the coface and codegeneracy maps arising in a standard manner from the unit $S^{0} \rightarrow R$ and the multiplication $R \wedge R \rightarrow R$. Under good conditions, for a spectrum $X$, the cosimplicial diagram

$$
R^{\bullet}(X) \stackrel{\text { def }}{=} X \wedge \mathrm{CB}^{\bullet}(R)=\{X \wedge R \rightarrow \underset{\rightarrow}{\rightarrow} \wedge R \wedge R \underset{\rightarrow}{\rightarrow} \cdots\}
$$

will be a resolution of $X$ in the sense that the natural map $X \rightarrow \operatorname{Tot}\left(R^{\bullet}(X)\right)$ is an equivalence. If so, then one has a homotopy spectral sequence

$$
E_{2}^{s, t}=\pi^{s} \pi_{t} R^{\bullet}(X) \Longrightarrow \pi_{t-s} X .
$$

If $R=M U$ is complex bordism, and $X$ is connective, then the associated spectral sequence is the Adams-Novikov spectral sequence.

Suppose now that $R$ is also homotopy commutative, and for each $s, \pi_{*} R^{\wedge(s+1)}$ is concentrated in even degrees. Then we get a cosimplicial commutative $\operatorname{ring} \pi_{*} R^{\wedge(s+1)}$, over which $\pi_{*}\left(R^{\wedge s+1} \wedge X\right)$ is a cosimplicial module. If $R_{*} R$ is flat over $R_{*}$, the diagram

$$
\pi_{*} R \rightarrow \pi_{*}(R \wedge R) \stackrel{\rightarrow}{\rightarrow} \ldots
$$

is determined by its 2-truncation, and it is a commutative Hopf algebroid. This presents a stack $\mathfrak{X}$. Furthermore, the cosimplicial $\pi_{*} R^{\bullet}$-module $\pi_{*} R^{\bullet}(X)$ defines a quasi-coherent sheaf on $\mathfrak{X}$, i.e., a comodule over the Hopf algebroid $\left(R_{*}, R_{*} R\right)$. We shall denote this by $\mathcal{F}(X)$.

The chain complex

$$
\pi_{*}(R \wedge X) \rightarrow \pi_{*}(R \wedge R \wedge X) \rightarrow \ldots
$$

whose cohomology is the $E_{2}$ page of (3), can be identified with the cobar complex

$$
R_{*}(X) \rightarrow R_{*}(R) \otimes_{R_{*}} R_{*} X \rightarrow \ldots,
$$

which computes the cohomology of the sheaf $\mathcal{F}(X)$. In particular, the spectral sequence (3) can be written as

$$
H^{s}(\mathfrak{X}, \mathcal{F}(X)) \Longrightarrow \pi_{t-s} X .
$$

It is convenient to make one further modification. The rings $R_{*}, R_{*} R$ are graded rings, and the sheaves $\mathcal{F}(X)$ come from graded comodules over the Hopf algebroid $\left(R_{*}, R_{*} R\right)$. Let us now suppose that $R_{*}, R_{*} R$ are evenly graded. The grading determines (and is equivalent to) a $\mathbb{G}_{m}$-action on the Hopf algebroid $\left(R_{*}, R_{*} R\right)$, or on the 
stack $\mathfrak{Y}$, such that an element in degree $2 k$ is acted on by $\mathbb{G}_{m}$ with eigenvalue given by the character $\chi_{k}: \mathbb{G}_{m} \rightarrow \mathbb{G}_{m}, \chi_{k}(u)=u^{k}$. We can regard $\mathcal{F}(X)$ as the sum of comodules $\mathcal{F}_{\text {even }}(X) \oplus \mathcal{F}_{\text {odd }}(X)$, where each of the two summands inherits a $\mathbb{G}_{m}$-action in a similar manner. That is, $\mathcal{F}_{\text {even }}(X)$ is given a $\mathbb{G}_{m}$-action in the same manner, and $\mathbb{G}_{m}$ acts on $\mathcal{F}_{2 k+1}(X)$ by the character $\chi_{k}$.

If we form the stack $\mathfrak{Y}=\mathfrak{X} / \mathbb{G}_{m}$, then $\mathfrak{Y}$ comes with a tautological line bundle $\omega$, corresponding to the $\left(R_{*}, R_{*} R\right)$-comodule $R_{*+2}$. Moreover, $\mathcal{F}_{\text {even }}$ and $\mathcal{F}_{\text {odd }}$ descend to functors into $\operatorname{Mod}(\mathfrak{Y})$, which is equivalent to the category of evenly graded comodules over $\left(R_{*}, R_{*} R\right)$. The spectral sequence can be written

$$
E_{2}^{s, t} \Longrightarrow \pi_{t-s} X, \quad E_{2}^{s, t}= \begin{cases}H^{s}\left(\mathfrak{Y}, \mathcal{F}_{\text {even }}(X) \otimes \omega^{t^{\prime}}\right) & \text { if } t=2 t^{\prime} \\ H^{s}\left(\mathfrak{Y}, \mathcal{F}_{\text {odd }}(X) \otimes \omega^{t^{\prime}}\right) & \text { if } t=2 t^{\prime}+1 .\end{cases}
$$

We now recall Quillen's theorem.

Definition 2.1. $M_{F G}$ is the moduli stack of formal groups. In other words, $M_{F G}$ is the 2-functor $M_{F G}$ : Ring $\rightarrow \mathbf{G p d}$ assigning to any commutative ring $A$ the groupoid of formal group schemes $X \rightarrow \operatorname{Spec}(A)$ which are Zariski locally (on $A$ ) isomorphic to $\operatorname{Spf}(A[[x]])$ as pointed formal schemes.

When $R=M U$ (as we will henceforth assume), Quillen's theorem states that there is an equivalence between the stack that one builds from the evenly graded Hopf algebroid $\left(M U_{*}, M U_{*} M U\right)$ and the moduli stack of formal groups.

We can often describe the quasi-coherent sheaves $\mathcal{F}_{\text {even }}(X), \mathcal{F}_{\text {odd }}(X)$ on $M_{F G}$ in terms of the geometry of formal groups.

Example 2.2. The line bundle $\omega$ described above on $M_{F G}$ (that is, associated to the $\left(M U_{*}, M U_{*} M U\right)$-comodule $M U_{*+2}$, which arises topologically from $\left.S^{-2}\right)$ assigns to a formal group $X$ the cotangent space $\mathcal{O}_{X}(-e) / \mathcal{O}_{X}(-2 e)$ of functions on $X$ that vanish at zero, modulo functions that vanish to order two (that is, the dual to the Lie algebra).

\subsection{Stacks associated to ring spectra}

We will also need a means of extracting stacks from ring spectra which may not be as well-behaved as $M U$ (cf. [7, Ch. 7]). Suppose $X$ is a homotopy commutative ring spectrum. Then the above cosimplicial diagram

$$
X \wedge M U \stackrel{\rightarrow}{\rightarrow} X \wedge M U \wedge M U \stackrel{\rightarrow}{\rightarrow} \cdots
$$

is a diagram of ring spectra itself, and the associated diagram of homotopy groups is a diagram of commutative rings if $M U_{*} X$ is evenly graded. Consequently, the associated sheaf $\mathcal{F}(X)=\mathcal{F}_{\text {even }}(X)$ on the stack $M_{F G}$ is a sheaf of commutative rings, and can be used to present another stack, affine over $M_{F G}$.

Definition 2.3. We write $\operatorname{Stack}(X)$ for the stack built in the above manner. Equivalently, $\operatorname{Stack}(X)$ is the stack associated to the Hopf algebroid $\left(M U_{*} X, M U_{*}(M U \wedge X)\right)$.

Example 2.4. Consider $X=M U$. Recall that $\operatorname{Spec}\left(\pi_{*}(M U \wedge M U)\right)$ classifies a pair of formal group laws with a strict isomorphism between them. The scheme $\operatorname{Spec}\left(\pi_{*}(M U \wedge M U \wedge M U)\right)$ corresponds to a triple of formal group laws with strict 
isomorphisms between them. It follows that the associated stack is equivalent to the $\mathbb{G}_{m}$-quotient of $\operatorname{Spec}(L)$, where $L$ is the Lazard ring. More generally, whenever $R$ is a complex-orientable ring spectrum with $\pi_{*} R$ evenly graded, the stack $\operatorname{Stack}(R)$ is the $\mathbb{G}_{m}$-quotient of $\operatorname{Spec}\left(R_{*}\right)$. It follows in particular that for such $R$, the sheaf $\mathcal{F}(R)$ is the push-forward of the structure sheaf under the map $\operatorname{Spec}\left(R_{*}\right) / \mathbb{G}_{m} \rightarrow M_{F G}$.

\subsection{Even periodic ring spectra}

Let $X$ be a spectrum. As we saw, $X$ defines quasi-coherent sheaves $\mathcal{F}_{\text {odd }}(X)$, $\mathcal{F}_{\text {even }}(X)$ on the moduli stack $M_{F G}$ of formal groups. These come from $M U_{*}(X)$ together with the comodule structure over the Hopf algebroid $\left(M U_{*}, M U_{*} M U\right)$ and the grading. Equivalently, we can consider the periodic complex bordism spectrum $M P=\bigvee_{i \in \mathbb{Z}} \Sigma^{2 i} M U$. In this case, we can write $M U_{*}(X)=M P_{0}(X) \oplus M P_{1}(X)$ and the even and odd parts of the grading correspond to the two summands.

On the flat site of $M_{F G}$, there is a classical topological interpretation of these sheaves.

Definition $2.5([1])$. A homotopy commutative ring spectra $E$ is even periodic if $\pi_{i} E=0$ for $i$ odd, and if $\pi_{2} E$ is an invertible module over $\pi_{0} E$ with the property that the multiplication map $\pi_{2} E \otimes_{\pi_{0} E} \pi_{-2} E \rightarrow \pi_{0} E$, is an isomorphism.

Given an even periodic ring spectrum $E$, the formal scheme $\operatorname{Spf} E^{0}\left(\mathbb{C P}^{\infty}\right)$ is a formal group over $E_{0}$ (see [1]), and is classified by a morphism $q: \operatorname{Spec}\left(E_{0}\right) \rightarrow M_{F G}$. If $q: \operatorname{Spec}\left(E_{0}\right) \rightarrow M_{F G}$ is flat, then $E$ is called a Landweber-exact theory and one has functorial isomorphisms:

$$
q^{*}\left(\omega^{j} \otimes \mathcal{F}_{\text {even }}(X)\right) \simeq E_{2 j}(X), \quad q^{*}\left(\omega^{j} \otimes \mathcal{F}_{\text {odd }}(X)\right) \simeq E_{2 j+1}(X) .
$$

In particular, for a Landweber-exact theory, $E_{*}(X)$ can be recovered from the sheaves $\mathcal{F}_{\text {even }}(X), \mathcal{F}_{\text {odd }}(X)$. The stack associated to the ring spectrum $E$ is precisely $\operatorname{Spec}\left(E_{0}\right)$.

Conversely, given a ring $R$ and a flat morphism $q$ : $\operatorname{Spec}(R) \rightarrow M_{F G}$, the functor

$$
X \mapsto \bigoplus_{j} q^{*}\left(\omega^{j} \otimes\left(\mathcal{F}_{\text {even }}(X) \oplus \mathcal{F}_{\text {odd }}(X)\right)\right)
$$

defines a multiplicative homology theory, representable by a Landweber-exact ring spectrum. The result is a presheaf of multiplicative homology theories on the flat site of $M_{F G}$. One reason this point of view is so useful is the following criterion for flatness over $M_{F G}$ given by the Landweber exact functor theorem. For a discussion of the Landweber exact functor theorem and its interpretation via the language of stacks (due to Hopkins), we refer to [24] and [20, Lecture 16].

\subsection{Topological modular forms}

Although $M_{F G}$ is a very large stack, there are smaller stacks that can be used to approximate it.

Definition 2.6. Let $M_{\overline{e l l}}$ be the moduli stack of generalized elliptic curves. In other words, $M_{\overline{e l l}}$ assigns to each commutative ring $R$ the groupoid of all pairs $(\pi: C \rightarrow$ $\operatorname{Spec}(R), e: \operatorname{Spec}(R) \rightarrow C)$, where $\pi, e$ are such that:

1. $\pi$ is a proper, flat morphism of finite presentation. 
2. The fibers of $\pi$ have arithmetic genus one, and they are either smooth curves or curves with a single nodal singularity.

3. $e$ is a section of $\pi$ whose image is contained in the smooth locus of $\pi$.

There is a flat map $M_{\overline{e l l}} \rightarrow M_{F G}$ which sends each generalized elliptic curve to its formal completion at the identity, which acquires the structure of a formal group. Consequently, any spectrum $X$ defines by pullback quasi-coherent sheaves on $M_{\overline{e l l}}$ as well; we will denote these too by $\mathcal{F}_{\text {even }}(X), \mathcal{F}_{\text {odd }}(X)$.

As before, the quasi-coherent sheaf $\mathcal{F}(X)$ on $M_{\overline{e l l}}$ has a topological interpretation. Given a flat map $q: \operatorname{Spec}(R) \rightarrow M_{\overline{e l l}}$ classifying an elliptic curve $C \rightarrow \operatorname{Spec}(R)$, one can construct an elliptic spectrum $E$ : in other words, $E$ is even periodic with $E_{0}=R$, and there is an isomorphism of formal groups

$$
\operatorname{Spf} E^{0}\left(\mathbb{C P}^{\infty}\right) \simeq \hat{C},
$$

between the formal group of $E$ and the formal completion of $C$. In this case, one has $E_{0}(X) \simeq q^{*}\left(\mathcal{F}_{\text {even }}(X)\right)$ and $E_{1}(X) \simeq q^{*}\left(\mathcal{F}_{\text {odd }}(X)\right)$. Moreover, there is a functorial isomorphism

$$
E_{2 j}(*) \simeq \omega^{j}, \quad E_{2 j+1}(*)=0,
$$

where $\omega$ is the line bundle on $M_{\overline{\text { ell }}}$ obtained by pulling back $\omega$ on $M_{F G}$.

The work of Goerss, Hopkins, Miller, and Lurie shows that, when we restrict to étale affines over $M_{\overline{e l l}}, E$ actually can be taken to be an $E_{\infty}$-ring spectrum, and that the above construction is can be made to be functorial: it defines a sheaf $\mathcal{O}^{\text {top }}$ of $E_{\infty}$-ring spectra on the étale site of $M_{\overline{e l l}}$. We refer to

Theorem 2.7 (Goerss, Hopkins, Miller, Lurie).

1. (Existence) There is a sheaf $\mathcal{O}^{\text {top }}$ of $E_{\infty}$-ring spectra on the étale site of $M_{\overline{e l l}}$, such that for $\operatorname{Spec}(R) \rightarrow M_{\overline{e l l}}$ an affine étale open classifying an elliptic curve $C \rightarrow \operatorname{Spec}(R)$, the $E_{\infty}$-ring $\mathcal{O}^{\text {top }}(\operatorname{Spec}(R))$ is an elliptic spectrum corresponding to $C$.

2. (Gap theorem) Moreover, $\pi_{j}\left(\Gamma\left(M_{\overline{e l l}}, \mathcal{O}^{\text {top }}\right)\right)=0$ for $-21<j<0$.

We refer to [7, Lectures 11-12] for a treatment of the construction of the sheaf $\mathcal{O}^{\text {top }}$ and to [4] and [7, Lecture 13] for some of the computations of the homotopy groups. See also the masters thesis of Konter [17] for the latter.

They define

$$
\operatorname{Tmf}=\Gamma\left(M_{\overline{e l l}}, \mathcal{O}^{\text {top }}\right), \quad \operatorname{tmf}=\tau_{\geqslant 0}(\operatorname{Tmf}),
$$

where $\tau_{\geqslant 0}$ denotes the connective cover. In other words, Tmf is the $E_{\infty}$-ring spectrum of global sections of $\mathcal{O}^{\text {top }}$, constructed as a homotopy limit of the associated elliptic spectra as $\operatorname{Spec}(R) \rightarrow M_{\overline{e l l}}$ ranges over étale morphisms. Since Tmf is constructed as a homotopy limit, we have a descent spectral sequence

$$
H^{i}\left(M_{\overline{e l l}}, \pi_{2 j} \mathcal{O}^{\text {top }}\right)=H^{i}\left(M_{\overline{e l l}}, \omega^{j}\right) \Longrightarrow \pi_{2 j-i} \operatorname{Tmf} .
$$

More generally, if $X$ is a spectrum, then we get a spectral sequence

$$
H^{i}\left(M_{\overline{e l l}}, \pi_{j}\left(\mathcal{O}^{\text {top }} \wedge X\right)\right) \Longrightarrow \pi_{j-i}(\operatorname{Tmf} \wedge X) .
$$

Here we use the fact that $\operatorname{Tmf} \wedge X \simeq \Gamma\left(M_{\overline{e l l}}, \mathcal{O}^{\text {top }} \wedge X\right)$; the fact that this holds for any $X$, not necessarily finite, follows because $M_{\overline{e l l}} \rightarrow M_{F G}$ is tame (cf. [22, Th. 4.14 
and 7.2]). A knowledge of $\mathcal{F}_{\text {even }}(X)$ and $\mathcal{F}_{\text {odd }}(X)$, or equivalently of $\pi_{0}\left(\mathcal{O}^{\text {top }} \wedge X\right)$ and $\pi_{1}\left(\mathcal{O}^{\text {top }} \wedge X\right)$, is the information necessary to identify the $E_{2}$-page of the spectral sequence.

The following case, which is that of interest to us, offers a simplification of the spectral sequence:

Definition $2.8([1])$. A connective spectrum $X$ is even if $H_{*}(X ; \mathbb{Z})$ is free and concentrated in even dimensions. We can make a similar definition for a $p$-local spectrum, for a prime $p$.

In the even case, the sheaf $\mathcal{F}_{\text {even }}(X)$ on $M_{F G}$ can be interpreted in the following way: for a flat morphism $q$ : $\operatorname{Spec}(R) \rightarrow M_{F G}$, the $R$-module $q^{*} \mathcal{F}_{\text {even }}(X)$ is identified with $E_{0}(X)$ for $E$ the Landweber-exact, even-periodic ring spectrum associated with $q$. It follows from the (degenerate) Atiyah-Hirzebruch spectral sequence that $E_{1}(X)=0$, and in particular $\mathcal{F}_{\text {odd }}(X)=0, \mathcal{F}(X)=\mathcal{F}_{\text {even }}(X)$. We find that $\mathcal{F}(X)$ can be simply viewed as a sheaf on $M_{F G}$ or $M_{\overline{e l l}}$. In this case, the Adams-Novikov spectral sequence can be written as

$$
H^{i}\left(M_{F G}, \mathcal{F}(X) \otimes \omega^{j}\right) \Longrightarrow \pi_{2 j-i}(X)
$$

and the descent spectral sequence can be written as

$$
H^{i}\left(M_{\overline{e l l}}, \omega^{j} \otimes \mathcal{F}(X)\right) \Longrightarrow \pi_{2 j-i}(\operatorname{Tmf} \wedge X) .
$$

\section{A vector bundle on $M_{c u b}$}

In this section, we will introduce the moduli stack $M_{c u b}$ of cubic curves and exhibit an eight-dimensional vector bundle at the prime 2 .

\subsection{The stack $M_{c u b}$}

For our purposes, it will be convenient to work over the larger stack of all cubic curves. The results on cubic curves that we need can be found in [5].

Definition 3.1. Given a scheme $S$, a cubic curve over $S$ is a map $p: E \rightarrow S$ which is flat and proper of finite presentation, together with a section $e: S \rightarrow E$ whose image is contained in the smooth locus of $p$. The geometric fibers of $p$ are required to be reduced, irreducible curves of arithmetic genus one. We denote by $M_{c u b}$ the stack which assigns to each commutative ring $R$ the groupoid of cubic curves over $\operatorname{Spec}(R)$. Below, we will recall a Hopf algebroid presentation of $M_{c u b}$.

Definition 3.2. There is a line bundle $\omega$ on the stack $M_{c u b}$, which assigns to a cubic curve $p: C \rightarrow \operatorname{Spec}(R), e: \operatorname{Spec}(R) \rightarrow C$ the $R$-module of sections of $\mathcal{O}_{C}(-e) / \mathcal{O}_{C}(-2 e)$ : that is, the cotangent space along the zero section $e$, or the dual to the Lie algebra.

If $p: E \rightarrow S$ is a cubic curve, there are three possibilities for each geometric fiber: it can be an elliptic curve, a nodal cubic in $\mathbb{P}^{2}$, or a cuspidal cubic in $\mathbb{P}^{2}$ (isomorphic to the projective closure of the curve defined by $\left.y^{2}=x^{3}\right)$. In the first two cases, there are no infinitesimal automorphisms. However, the multiplicative group $\mathbb{G}_{m}$ acts on the cuspidal curve. In particular, $M_{c u b}$ is only an Artin stack, which contains 
the Deligne-Mumford stack $M_{\overline{e l l}}$ as an open substack. The complement of $M_{\overline{e l l}}$ in $M_{c u b}$ is given by the vanishing locus of the modular forms $c_{4} \in H^{0}\left(M_{c u b}, \omega^{4}\right)$ and $\Delta \in H^{0}\left(M_{c u b}, \omega^{12}\right)$ (cf. [5] for expressions for these).

Zariski locally on $S$, a cubic curve can be described as a subscheme of $\mathbb{P}_{S}^{2}$ cut out by a cubic equation

$$
y^{2}+a_{1} x y+a_{3} y=x^{3}+a_{2} x^{2}+a_{4} x+a_{6} .
$$

In order to show this, one has to choose coordinates $x, y$ (which are chosen as sections of appropriate line bundles). Consequently, one can write down an explicit presentation of this stack via a Hopf algebroid. We sketch this below.

Let $E \rightarrow \operatorname{Spec}(R)$ be a cubic curve, given by a cubic equation (5). Given one choice of $x, y$, the collection of other choices of coordinates is parametrized by

$$
\begin{aligned}
& x=u^{2} x^{\prime}+r, \\
& y=u^{3} y^{\prime}+s u^{2} x^{\prime}+t,
\end{aligned}
$$

where $u \in R^{*}$ and $r, s, t \in R$. These are the isomorphisms between Weierstrass curves.

In particular, we can represent $M_{c u b}$ as a Hopf algebroid (the Weierstrass Hopf algebroid) over the ring $\mathbb{Z}\left[a_{1}, a_{2}, a_{3}, a_{4}, a_{6}\right]$. The left and right units come from the transformation laws of a cubic equation, and the comultiplication comes from composition of isomorphisms.

Suppose given a cubic curve (5), and suppose one makes a change of coordinates as in (6). Then the new Weierstrass cubic, in coordinates $x^{\prime}, y^{\prime}$, has the form

$$
y^{\prime 2}+a_{1}^{\prime} x^{\prime} y^{\prime}+a_{3}^{\prime} y^{\prime}=x^{\prime 3}+a_{2}^{\prime} x^{\prime 2}+a_{4}^{\prime} x^{\prime}+a_{6}^{\prime},
$$

where:

$$
\begin{gathered}
u a_{1}^{\prime}=a_{1}+2 s, \\
u^{2} a_{2}^{\prime}=a_{2}-s a_{1}+3 r-s^{2}, \\
u^{3} a_{3}^{\prime}=a_{3}+r a_{1}+2 t, \\
u^{4} a_{4}^{\prime}=a_{4}-s a_{3}+2 a_{2} r-(t+r s) a_{1}+3 r^{2}-2 s t, \\
u^{6} a_{6}^{\prime}=a_{6}+r a_{4}+r^{2} a_{2}+r^{3}-t a_{3}-t^{2}-r t a_{1} .
\end{gathered}
$$

The stack $M_{c u b}$ is presented either by the Hopf algebroid

$$
\left(\mathbb{Z}\left[a_{1}, \ldots, a_{6}\right], \mathbb{Z}\left[a_{1}, \ldots, a_{6}\right]\left[u^{ \pm 1}, r, s, t\right]\right),
$$

or by the graded Hopf algebroid

$$
\left(\mathbb{Z}\left[a_{1}, \ldots, a_{6}\right], \mathbb{Z}\left[a_{1}, \ldots, a_{6}\right][r, s, t]\right), \quad\left|a_{i}\right|=2 i \text { and }|r|=4,|s|=2,|t|=6 .
$$

The grading is doubled as in topology.

Given a cubic curve $E \rightarrow \operatorname{Spec}(R)$, there is induced a structure of commutative group scheme on the smooth locus $E^{\circ} \rightarrow \operatorname{Spec}(R)$ (see Proposition 2.7 of [6]). In fact, $E^{\circ}$ can be described as the relative Picard scheme $\operatorname{Pic}_{E / \operatorname{Spec}(R)}^{0}$; see Theorem 2.6 of [6]. This is also described in Proposition 2.5 of III in [30]. In particular, we can take the formal completion at the zero section and get a formal group over $R$. This gives a morphism of stacks

$$
M_{c u b} \rightarrow M_{F G} .
$$


Example 3.3. The geometric points of $M_{c u b}$ fall into four types in characteristic $p>0$. There are the ordinary elliptic curves, which map to height one formal groups, as do the nodal elliptic curves. There are supersingular elliptic curves, which map to height two formal groups. Finally, there is the cuspidal cubic, which maps to the additive formal group (of infinite height).

Consequently, given a spectrum $X$, defining sheaves $\mathcal{F}_{\text {even }}(X), \mathcal{F}_{\text {odd }}(X)$ on $M_{F G}$, we can pull back to define a sheaf (which we will still denote by the same notation) on $M_{\text {cub }}$. If $X$ is an even spectrum, then $\mathcal{F}(X)=\mathcal{F}_{\text {even }}(X)$ will be a vector bundle on $M_{c u b}$ too. Note, however, that the map $M_{c u b} \rightarrow M_{F G}$ is no longer flat, unlike the $\operatorname{map} M_{\overline{e l l}} \rightarrow M_{F G}$.

We note that the line bundle $\omega$ on $M_{F G}$ pulls back to the line bundle $\omega$ on $M_{\text {cub }}$; given an elliptic curve $p: C \rightarrow \operatorname{Spec}(R), e: \operatorname{Spec}(R) \rightarrow C$, the $R$-module $\mathcal{O}_{C}(-e) / \mathcal{O}_{C}(-2 e)$ is also the Lie algebra of the formal group. In particular, it can also be described as associated to the graded comodule $\mathbb{Z}\left[a_{1}, \ldots, a_{6}\right]$ over the Weierstrass Hopf algebroid with the grading shifted by 2 .

\subsection{An eight-fold cover of $M_{c u b}$}

Henceforth, in this section, we work localized at 2 throughout: in particular, stacks such as $M_{c u b}$ will really mean $M_{c u b} \times_{\operatorname{Spec}(\mathbb{Z})} \operatorname{Spec}\left(\mathbb{Z}_{(2)}\right)$. We will exhibit an eightdimensional vector bundle on $M_{c u b}$ (which we will later see corresponds to a finite spectrum) by producing a finite flat cover $p: T \rightarrow M_{c u b}$, for $T$ a simpler stack. The associated vector bundle will be $p_{*} \mathcal{O}_{T}$. We refer to Section 4.5 below for a discussion of the modular interpretation.

Namely, we take for $T$ the stack-theoretic quotient

$$
T=\operatorname{Spec}\left(\mathbb{Z}_{(2)}\left[\alpha_{1}, \alpha_{3}\right]\right) / \mathbb{G}_{m},
$$

where the $\mathbb{G}_{m}$-action corresponds to the grading of the ring $\mathbb{Z}_{(2)}\left[\alpha_{1}, \alpha_{3}\right]$ with $\left|\alpha_{1}\right|=$ $2,\left|\alpha_{3}\right|=6$, where the grading is doubled in accordance with topology. In other words, we can think of $T$ as the stack associated to the prestack sending a ring $R$ to the groupoid of pairs of elements $\left(\alpha_{1}, \alpha_{3}\right) \in R$, with

$$
\operatorname{Hom}\left(\left(\alpha_{1}, \alpha_{3}\right),\left(\alpha_{1}^{\prime}, \alpha_{3}^{\prime}\right)\right)=\left\{u \in R^{*}: u \alpha_{1}=\alpha_{1}^{\prime}, u^{3} \alpha_{3}=\alpha_{3}^{\prime}\right\} .
$$

To produce the cover $p: T \rightarrow M_{c u b}$, observe first that there is a map $\operatorname{Spec}\left(\mathbb{Z}_{(2)}\left[\alpha_{1}, \alpha_{3}\right]\right) \rightarrow M_{\text {cub }}$ classifying the cubic curve

$$
y^{2}+\alpha_{1} x y+\alpha_{3} y=x^{3} .
$$

Observe that $\mathbb{G}_{m}$ also acts on the cubic curve in a corresponding fashion as in the action on $\mathbb{Z}_{(2)}\left[\alpha_{1}, \alpha_{3}\right]$. Namely, given an invertible element $u$, we have the transformation $x \mapsto u^{2} x, y \mapsto u^{3} y$ from the curve $y^{2}+\alpha_{1} x y+\alpha_{3} y=x^{3}$ into the curve $y^{2}+u \alpha_{1} x y+u^{3} \alpha_{3} y=x^{3}$. These isomorphisms allow us to produce the morphism of stacks $T \rightarrow M_{c u b}$, as desired.

Proposition 3.4. The map $p: T \rightarrow M_{c u b}$ is representable and is a finite, flat cover of rank eight.

Proposition 3.4 will be proved in the next subsection. 


\subsection{Verification of the rank eight cover}

The goal of this subsection is to establish Proposition 3.4. To check that a morphism $\mathfrak{X} \rightarrow \mathfrak{Y}$ of stacks is finite and flat, we just need to show that for every map $\operatorname{Spec}(R) \rightarrow \mathfrak{Y}$, the pullback $\operatorname{Spec}(R) \times_{\mathfrak{Y}} \mathfrak{X}$ is a finite flat cover of $\operatorname{Spec}(R)$. In our case, we need to show that for every morphism $\operatorname{Spec}(R) \rightarrow M_{c u b}$, when one forms the pullback square,

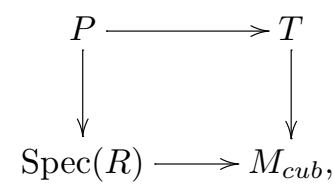

the fiber product $P$ is an affine scheme, corresponding to the Spec of an algebra which is a finite, flat $R$-module of rank eight.

Let us first identify the pullback $\operatorname{Spec}(R) \times_{M_{c u b}} \operatorname{Spec}\left(\mathbb{Z}_{(2)}\left[\alpha_{1}, \alpha_{3}\right]\right)$ concretely. Suppose that $\operatorname{Spec}(R) \rightarrow M_{c u b}$ classifies a cubic curve in $\mathbb{P}_{R}^{2}$ cut out by the Weierstrass equation

$$
y^{2}+a_{1} x y+a_{3} y=x^{3}+a_{2} x+a_{4}+a_{6}, \quad a_{i} \in R .
$$

By passing to a Zariski cover of $\operatorname{Spec}(R)$, we can always assume this. Then, to form the pullback of stacks, we have to consider the scheme parametrizing changes of coordinates (over $R$ ) which will transform this equation into an equation

$$
y^{\prime 2}+a_{1}^{\prime} x^{\prime} y^{\prime}+a_{3}^{\prime} y^{\prime}=x^{\prime 3}+a_{2}^{\prime} x^{\prime 2}+a_{4}^{\prime} x^{\prime}+a_{6}^{\prime},
$$

where $a_{2}^{\prime}, a_{4}^{\prime}, a_{6}^{\prime}=0$. In other words, looking back at the previous formulas, $\operatorname{Spec}(R) \times_{M_{\text {cub }}} \operatorname{Spec}\left(\mathbb{Z}_{(2)}\left[\alpha_{1}, \alpha_{3}\right]\right)$ is the scheme parametrizing elements $u, r, s, t$ (with $u$ invertible) satisfying the equations

$$
\begin{aligned}
& 0=a_{2}-s a_{1}+3 r-s^{2}, \\
& 0=a_{4}-s a_{3}+2 a_{2} r-(t+r s) a_{1}+3 r^{2}-2 s t, \\
& 0=a_{6}+r a_{4}+r^{2} a_{2}+r^{3}-t a_{3}-t^{2}-r t a_{1} .
\end{aligned}
$$

In other words, it is $R\left[u^{ \pm 1}, r, s, t\right]$ modulo the above relations. Taking the pullback $P=\operatorname{Spec}(R) \times_{M_{\text {cub }}} T$ corresponds to taking the $\mathbb{G}_{m}$-quotient: in other words, we just have to ignore $u$.

We find that $P$ is a closed subscheme of affine space $\mathbb{A}_{R}^{3}$ cut out by the three equations above; by the first relation, $r$ is determined in terms of $s$, and $P$ is even a closed subscheme of $\mathbb{A}_{R}^{2}$ cut out by two equations. Our goal is to show that it is finite flat over $\operatorname{Spec}(R)$, of rank eight. Let us first show finiteness.

Lemma 3.5. Let $R$ be a ring, and let $a_{1}, a_{2}, a_{3}, a_{4}, a_{6} \in R$. Then the quotient of $R[r, s, t]$ by the above three relations $(7)$ is a finite $R$-module.

Proof. We can do this in the universal case where $R=\mathbb{Z}_{(2)}\left[a_{1}, \ldots, a_{6}\right]$. Let $I$ be the ideal generated by the above relations. In this case, observe that $R$ is naturally a graded ring (where we set $\left|a_{i}\right|=2 i$ ), and the $\operatorname{ring} R[r, s, t] / I$ is a graded ring too, if we set

$$
|r|=4, \quad|s|=2, \quad|t|=6 .
$$

In other words, the relations defining $I$ are homogeneous. In view of Nakayama's lemma, we can now prove finiteness by taking the quotient by the augmentation 
ideal: that is, by working over $\mathbb{Z}_{(2)}$, and by setting all the $a_{i}=0$ (so that we have the cuspidal cubic $y^{2}=x^{3}$, and the grading of $R[r, s, t] / I$ comes from this grading). Then the relevant ring is the quotient of $\mathbb{Z}_{(2)}[r, s, t]$ by the relations

$$
3 r-s^{2}=0, \quad 3 r^{2}-2 s t=0, \quad r^{3}-t^{2}=0 .
$$

Alternatively, this is the quotient of $\mathbb{Z}_{(2)}[s, t]$ under the relations

$$
3\left(s^{2} / 3\right)^{2}=2 s t, \quad\left(s^{2} / 3\right)^{3}=t^{2} .
$$

Since this is a graded $\mathbb{Z}_{(2)}$-module each of whose graded pieces is finitely generated, we may as well prove finiteness after tensoring with $\mathbb{Z} / 2$, by the ungraded version of Nakayama's lemma. We then get the $\mathbb{Z} / 2$-algebra $\mathbb{Z} / 2[s, t] /\left(s^{4}, t^{2}\right)$, which is evidently finite over $\mathbb{Z} / 2$.

If $R$ is any ring, $a_{i} \in R$ for $i=1,2, \ldots, 6$, and we form the quotient $R[r, s, t] / I$ as above, the above proof also shows that there is a set of eight generators of the $R$-module $R[r, s, t] / I$, given by

$$
\left\{1, s, s^{2}, s^{3}, t, s t, s^{2} t, s^{3} t\right\} .
$$

Namely, we just need to prove this in the universal case $\mathbb{Z}_{(2)}\left[a_{1}, a_{2}, a_{3}, a_{4}, a_{6}\right]$, which reduces by the Nakayama-type argument above to the case of the cuspidal cubic over $\mathbb{Z} / 2$. We have seen that the above elements generate in that case and in fact form a basis.

Now that we know that the map $T \rightarrow M_{\text {cub }}$ is finite, flatness is automatic. In fact, we saw in the proof that for any map $\operatorname{Spec}(R) \rightarrow M_{c u b}$, the pullback $T \times{ }_{M_{c u b}} \operatorname{Spec}(R)$ was, up to Zariski localizing on $R$, of the form $R[r, s, t] / I$, where $I$ was an ideal generated by three elements. This implies flatness by the following lemma.

Lemma 3.6. Let $X$ be a noetherian scheme, and let $Z \subset \mathbb{A}_{X}^{n}$ be a closed subscheme locally cut out by $n$ equations. Suppose the fibers $Z_{x}, x \in X$ are zero-dimensional. Then $Z \rightarrow X$ is flat.

Proof. In fact, we may suppose $X=\operatorname{Spec}(R)$, for $(R, \mathfrak{m})$ a local noetherian ring. We need to show that the local rings $\mathcal{O}_{Z, z}$ are flat $R$-modules for each $z \in Z$ lying over the maximal ideal of $R$. The ring $\mathcal{O}_{Z, z}$ is obtained from a localization of $R\left[t_{1}, \ldots, t_{n}\right]$ at a maximal ideal $\mathfrak{M} \supset \mathfrak{m}$, by taking the quotient by $n$ equations $f_{1}, \ldots, f_{n}$. Observe moreover that the local ring $S=R\left[t_{1}, \ldots, t_{n}\right]_{\mathfrak{M}}$ is flat over $R$, and $S /\left(f_{1}, \ldots, f_{n}\right)$ is the localization of a finite $R$-module. Let $k$ be the residue field of $R$. Then $S \otimes_{R} k$ is an $n$-dimensional regular local ring and $\left(S \otimes_{R} k\right) /\left(f_{1}, \ldots, f_{n}\right)$ is artinian, so $f_{1}, \ldots, f_{n}$ form a regular sequence on $S \otimes_{R} k$. It follows now from a Nakayama argument (cf. [11, Prop. 15.1.16]) that $f_{1}, \ldots, f_{n}$ are regular on $S$ itself, and that $\mathcal{O}_{Z, z}=S /\left(f_{1}, \ldots, f_{n}\right)$ is a flat $R$-module.

Finally, let us show that the map $p: \operatorname{Spec}\left(\mathbb{Z}_{(2)}\left[\alpha_{1}, \alpha_{3}\right]\right) / \mathbb{G}_{m} \rightarrow M_{c u b}$ is a cover, i.e., that it is surjective. Surjectivity follows because $p$ is finite flat, so that the image is both open and closed; however, $M_{c u b}$ admits a cover by $\operatorname{Spec}\left(\mathbb{Z}_{(2)}\left[a_{1}, a_{2}, a_{3}, a_{4}, a_{6}\right]\right)$ and is thus connected.

The upshot of all this is that the map $p: T=\operatorname{Spec}\left(\mathbb{Z}_{(2)}\left[\alpha_{1}, \alpha_{3}\right]\right) / \mathbb{G}_{m} \rightarrow M_{c u b}$ is an eight-fold flat cover, and consequently the push-forward of the structure sheaf gives 
us an eight-dimensional vector bundle $\mathcal{V}$ on $M_{\text {cub }}$. Our goal is to show that this vector bundle is realized by an 8-cell complex.

\section{Calculation of $\operatorname{Tmf}_{*}(D A(1))$}

In this section, we recall the 2-local complex $D A(1)$ and show that the vector bundle it induces on $M_{\overline{e l l}}$ is the one constructed algebraically in the previous section. We will do this by producing a map $\mathcal{F}(D A(1)) \rightarrow \mathcal{F}(M U)$ and a map from $\mathcal{F}(M U)$ to the vector bundle of the previous section, over $M_{c u b}$. We will check that the composite is an isomorphism over the cuspidal cubic over $\mathbb{Z} / 2$. This analysis leads to the computation of $\operatorname{Tmf}_{*}(D A(1))$ by the descent spectral sequence. In the final subsection, we will discuss the analog at odd primes.

\subsection{The complex $D A(1)$}

In this section, we describe a 2-local complex with eight-dimensional homology.

We start by describing the question mark complex, a variant of which is constructed in [15, Lem. 7.2]. First, let $\nu: S^{3} \rightarrow S^{0}$ and $\eta: S^{1} \rightarrow S^{0}$ be the usual Hopf maps. We have $\eta \nu=0$. We draw the cofiber sequence for $\eta$, which runs $S^{1} \stackrel{\eta}{\rightarrow} S^{0} \rightarrow \Sigma^{-2} \mathbb{C P}^{2} \rightarrow$ $S^{2} \rightarrow \ldots$, and consider the diagram:

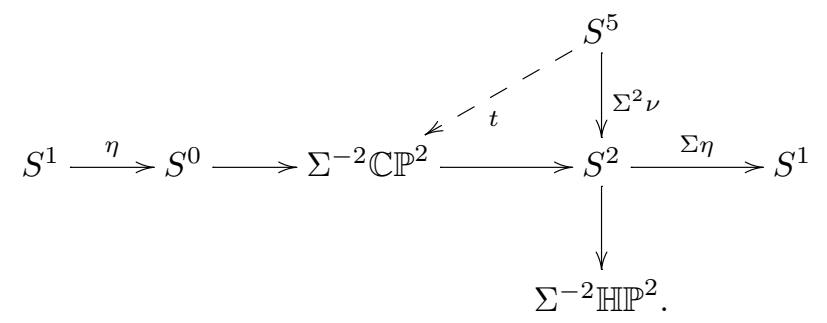

The map $t$ drawn as a dotted arrow exists because $\eta \nu=0$; it is even unique as $\pi_{5}\left(S^{0}\right)=0$.

Definition 4.1. The question mark complex $Q$ is defined to be the cofiber of $t$.

A simple calculation shows that $H^{*}(Q ; \mathbb{Z} / 2)$ is three-dimensional, with a basis given by the element $x_{0}$ in degree zero, and $\mathrm{Sq}^{2} x_{0}$ and $\mathrm{Sq}^{4} \mathrm{Sq}^{2} x_{0}$.

Definition 4.2. Let $\mathbb{D} Q=F\left(Q, S^{0}\right)$ be the Spanier-Whitehead dual to $Q$. We define the (2-local) complex $D A(1)$ to be the six-fold suspension of the cofiber of the coevaluation map $S^{0} \rightarrow Q \wedge \mathbb{D} Q$.

The composite of the coevaluation and evaluation maps $S^{0} \rightarrow Q \wedge \mathbb{D} Q \rightarrow S^{0}$ is multiplication by the Euler characteristic $\chi(Q)=3$. Since we are working 2-locally, we find that there is a splitting $Q \wedge \mathbb{D} Q \simeq S^{0} \vee \Sigma^{-6} D A(1)$.

Note that $D A(1)$ is an even 2-local spectrum. A computation shows that the cohomology $H^{*}(D A(1) ; \mathbb{Z} / 2)$ is a free module over the eight-dimensional algebra $D \mathcal{A}(1) \subset$ $\mathcal{A} / \mathcal{A S q}{ }^{1} \mathcal{A}$ generated by $\mathrm{Sq}^{2}, \mathrm{Sq}^{4} ;$ the cohomology is drawn in Figure 1. Its homology, as a comodule over $\mathcal{A}_{*}$, can be described as $\mathbb{Z} / 2\left\{1, \xi_{1}^{2}, \xi_{1}^{4}, \xi_{1}^{6}\right\} \otimes \mathbb{Z} / 2\left\{1, \zeta_{2}^{2}\right\} \subset \mathcal{A}_{*}$. 


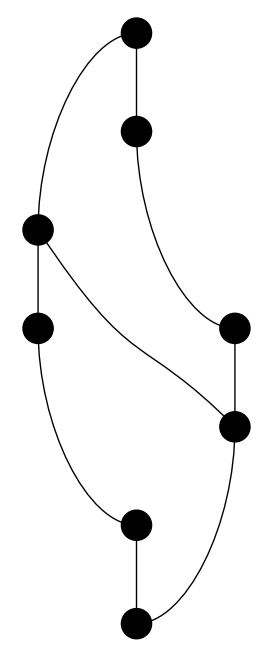

Figure 1: The cohomology of $D A(1)$. This depicts only the action of $\mathrm{Sq}^{2}, \mathrm{Sq}^{4}$; there is also a nontrivial $\mathrm{Sq}^{8}$ which is not shown

Remark 4.3. The complex is so named because its cohomology is free over the subalgebra of the Steenrod algebra generated by $\mathrm{Sq}^{2}$ and $\mathrm{Sq}^{4}$, which doubles the subalgebra $\mathcal{A}(1) \subset \mathcal{A}$ generated by $\mathrm{Sq}^{1}, \mathrm{Sq}^{2}$.

Since $D A(1)$ is an even spectrum, the Atiyah-Hirzebruch spectral sequence for $M U^{*}(D A(1))$ degenerates, and we can find a morphism $D A(1) \rightarrow M U$ which induces an isomorphism on $\pi_{0}$. This map is not unique, but we have specified its image in mod two homology after composing with the projection $M U \rightarrow B P$. To see this, we need to first recall some notation.

Convention. We let $\mathcal{A}_{*}=\pi_{*}(H \mathbb{Z} / 2 \wedge H \mathbb{Z} / 2)$ denote the $(\bmod 2)$ dual Steenrod algebra. Then we have

$$
\mathcal{A}_{*}=\mathbb{Z} / 2\left[\xi_{1}, \xi_{2}, \ldots\right], \quad\left|\xi_{i}\right|=2^{i}-1
$$

using the standard notation (cf. [26, Ch. 6] for a textbook reference). We will let $\zeta_{i}$ denote the Hopf conjugate of $\xi_{i}$.

We recall that the map $B P \rightarrow H \mathbb{Z} / 2$ induces an injection in mod 2 homology, and we get $H_{*}(B P ; \mathbb{Z} / 2)=\mathbb{Z} / 2\left[\xi_{i}^{2}\right]=\mathbb{Z} / 2\left[\zeta_{i}^{2}\right] \subset \mathcal{A}_{*}$. Compare [28, Ch. 4] for a textbook reference. The image of $H_{*}(D A(1) ; \mathbb{Z} / 2)$ in $H_{*}(B P ; \mathbb{Z} / 2)$ is spanned by $\left\{\xi_{1}^{a} \zeta_{2}^{b}\right\}$ for $0 \leqslant a \leqslant 3$ and $0 \leqslant b \leqslant 1$.

Both $D A(1)$ and $M U$ define quasi-coherent sheaves on $M_{F G}$, and thus on $M_{\overline{e l l}}$ and even $M_{c u b}$; these are denoted $\mathcal{F}(D A(1))$ and $\mathcal{F}(M U)$. The map $D A(1) \rightarrow M U$ defines an injection of sheaves

$$
\mathcal{F}(D A(1)) \rightarrow \mathcal{F}(M U)
$$

Our goal will be to produce a map $\mathcal{F}(M U) \rightarrow \mathcal{V}$, for $\mathcal{V}$ the eight-dimensional vector bundle constructed in the previous section, such that the composite is an isomorphism. We will construct the map, and check that it is an isomorphism on the cuspidal curve over $\mathbb{Z} / 2$. 


\subsection{Construction of a map}

Let us start by describing the sheaf $\mathcal{F}(M U)$ on the stack $M_{F G}$. This assigns to a formal group over a ring $R$ the ring parametrizing coordinates on this formal group, modulo action of $\mathbb{G}_{m}$. Namely, let $M_{F G}^{\text {coord }}$ be the moduli stack of formal groups together with a coordinate (i.e., an isomorphism of formal schemes with the formal affine line $\widehat{\mathbb{A}^{1}}$ ), so that $M_{F G}^{\text {coord }}$ is simply the spectrum of the Lazard ring. It parametrizes formal group laws (and no isomorphisms).

There is a $\mathbb{G}_{m}$-action on $M_{F G}^{\text {coord }}$, corresponding to twisting a coordinate: this induces the usual grading of the Lazard ring. There is a morphism of stacks

$$
q: M_{F G}^{\text {coord }} / \mathbb{G}_{m} \rightarrow M_{F G} .
$$

Then $\mathcal{F}(M U)=q_{*}(\mathcal{O})$.

Consider next the pullback diagram

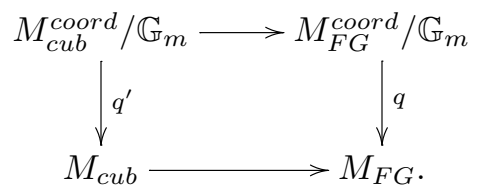

Here $M_{\text {cub }}^{\text {coord }} / \mathbb{G}_{m}$ is the stack parametrizing cubic curves together with a coordinate on the formal group, modulo $\mathbb{G}_{m}$-action. We have:

Theorem 4.4 (Cf. [16, Cor. 8.9]). There is an equivalence of stacks

$$
M_{\text {cub }}^{\text {coord }} / \mathbb{G}_{m}=\operatorname{Spec}\left(\mathbb{Z}_{(2)}\left[a_{1}, \ldots, a_{6}, e_{4}, e_{5}, \ldots\right]\right) / \mathbb{G}_{m} .
$$

The $\left\{a_{i}\right\}$ are the choice of coefficients for a Weierstrass equation. In fact, the choice of a coordinate modulo degree five on the formal group of a cubic curve is equivalent to the choice of a Weierstrass equation, and the remaining $e_{i}$ allow one to modify the coordinate in higher degrees.

We next construct a map $g: T=\operatorname{Spec}\left(\mathbb{Z}_{(2)}\left[\alpha_{1}, \alpha_{3}\right]\right) / \mathbb{G}_{m} \rightarrow M_{c u b}^{\text {coord }} / \mathbb{G}_{m}$ giving a commutative diagram

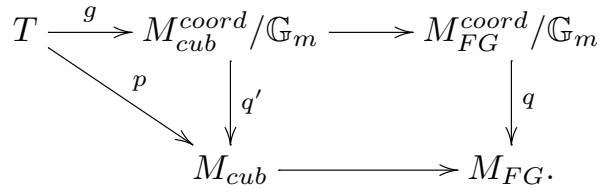

We define a map $g: T \rightarrow M_{c u b}^{\text {coord }} / \mathbb{G}_{m}$ by sending the cubic curve $y^{2}+\alpha_{1} x y+\alpha_{3} y=$ $x^{3}$ to the same cubic curve with the canonical coordinate $-x / y$. In other words, $a_{1} \mapsto \alpha_{1}, a_{3} \mapsto \alpha_{3}$, and all the other polynomial generators are mapped to zero. This morphism is a closed immersion.

We let $\mathcal{V}$ be the vector bundle of the previous section: that is, $\mathcal{V}=p_{*}(\mathcal{O})$. Then $\mathcal{V}$ is actually a bundle of finite, flat commutative algebras on $M_{c u b}$. The diagram naturally furnishes a map

$$
q_{*}^{\prime}(\mathcal{O}) \rightarrow q_{*}^{\prime}\left(g_{*}(\mathcal{O})\right) \simeq p_{*}(\mathcal{O})=\mathcal{V}
$$

which is a surjection of sheaves of algebras as $g$ was a closed immersion. Therefore, we know that $q_{*}^{\prime}(\mathcal{O}) \simeq \mathcal{F}(M U)$, and the map $\mathcal{F}(D A(1)) \rightarrow \mathcal{F}(M U)$ combined with the 
surjection $q_{*}^{\prime}(\mathcal{O}) \rightarrow \mathcal{V}$ induces a map $\mathcal{F}(D A(1)) \rightarrow \mathcal{V}$. This is a morphism of eightdimensional vector bundles on $M_{c u b}$.

\subsection{Identification of $\mathcal{F}(D A(1))$}

Our goal is to prove that the map $\mathcal{F}(D A(1)) \rightarrow \mathcal{V}$ constructed in the previous section is an isomorphism, or equivalently, that it is a surjection. The following lemma will be useful.

Lemma 4.5. Let $\mathcal{E} \rightarrow \mathcal{F}$ be a morphism of coherent sheaves on the stack $M_{\text {cub }}$ (localized at 2). Let $x: \operatorname{Spec}(\mathbb{Z} / 2) \rightarrow M_{\text {cub }}$ classify the cuspidal cubic curve. If $x^{*} \mathcal{E} \rightarrow x^{*} \mathcal{F}$ is a surjection of $\mathbb{Z} / 2$-vector spaces, then $\mathcal{E} \rightarrow \mathcal{F}$ is a surjection of coherent sheaves.

Proof. We can pull back the coherent sheaves to the flat cover $\operatorname{Spec}\left(\mathbb{Z}_{(2)}\left[a_{1}, a_{2}, a_{3}\right.\right.$, $\left.\left.a_{4}, a_{6}\right]\right)$ to obtain finitely generated graded modules $E, F$ over $\mathbb{Z}_{(2)}\left[a_{1}, a_{2}, a_{3}, a_{4}, a_{6}\right]$ and a map $E \rightarrow F$ such that

$$
E \otimes_{\mathbb{Z}_{(2)}\left[a_{1}, a_{2}, a_{3}, a_{4}, a_{6}\right]} \mathbb{Z} / 2 \rightarrow F \otimes_{\mathbb{Z}_{(2)}\left[a_{1}, a_{2}, a_{3}, a_{4}, a_{6}\right]} \mathbb{Z} / 2
$$

is a surjection. However, Nakayama's lemma, in both its graded and ungraded forms, now implies that $E \rightarrow F$ is a surjection of modules, as desired.

Remark 4.6. We note that if $\mathcal{F}$ is any quasi-coherent sheaf on $M_{c u b}$, then the $\mathbb{Z} / 2$ vector space $x^{*} \mathcal{F}$ has a canonical grading. This was used in the proof of Lemma 4.5, and it also can be interpreted as follows: the cuspidal curve $y^{2}=x^{3}$ admits a $\mathbb{G}_{m^{-}}$ action, and consequently all the vector spaces in question acquire a canonical $\mathbb{G}_{m^{-}}$ action (i.e., grading).

We can now prove the main result of this section.

Proposition 4.7. The composite map $\mathcal{F}(D A(1)) \rightarrow \mathcal{F}(M U) \simeq q_{*}^{\prime}(\mathcal{O}) \rightarrow \mathcal{V}$ is an isomorphism of vector bundles on $M_{\text {cub }}$ (in particular, on $\left.M_{\overline{e l l}}\right)$.

Proof. Since both bundles are eight-dimensional, it suffices to show that the map is a surjection. By Lemma 4.5, it suffices to show that if $x: \operatorname{Spec}(\mathbb{Z} / 2) \rightarrow M_{c u b}$ classifies the cuspidal cubic, then $x^{*} \mathcal{F}(D A(1)) \rightarrow x^{*} \mathcal{V}$ is a surjection of vector spaces.

Now, $D A(1)$ is an even 2-local spectrum: in particular, it has free $\mathbb{Z}_{(2)}$-homology and the AHSS degenerates for $M U_{*}(D A(1))$. This implies that

$$
x^{*}(\mathcal{F}(D A(1)))=M U_{*}(D A(1)) \otimes_{M U_{*}} \mathbb{Z} / 2 \simeq H_{*}(D A(1) ; \mathbb{Z} / 2) ;
$$

that is, we can get a description of $\mathcal{F}(D A(1))$ even over the cuspidal locus in terms of a homology theory. The same is true for $x^{*}(\mathcal{F}(M U)) \simeq H_{*}(M U ; \mathbb{Z} / 2)$. Note that the grading induced (cf. Remark 4.6) on the vector spaces $x^{*} \mathcal{F}(D A(1))\left(\right.$ resp. $x^{*} \mathcal{F}(M U)$ ) is simply the grading on homology.

Let us now describe the two maps

$$
H_{*}(D A(1) ; \mathbb{Z} / 2) \rightarrow H_{*}(M U ; \mathbb{Z} / 2), \quad H_{*}(M U ; \mathbb{Z} / 2) \rightarrow x^{*} \mathcal{V} .
$$

- First consider the map $H_{*}(D A(1) ; \mathbb{Z} / 2) \rightarrow H_{*}(M U ; \mathbb{Z} / 2)$. There is a unique nonzero indecomposable element $S$ in $H_{2}(M U ; \mathbb{Z} / 2)$ and a nonzero indecomposable element $T$ in $H_{6}(M U ; \mathbb{Z} / 2)$ with the property that

$$
\left\{1, S, S^{2}, S^{3}, T, S T, S^{2} T, S^{3} T\right\}
$$

forms a basis of the image of $H_{*}(D A(1) ; \mathbb{Z} / 2) \rightarrow H_{*}(M U ; \mathbb{Z} / 2)$. Under the 
reduction map $M U \rightarrow H \mathbb{Z} / 2$ and the induced map $H_{*}(M U ; \mathbb{Z} / 2) \rightarrow \mathcal{A}_{*}, S$ maps to $\xi_{1}^{2}$ and $T$ maps to $\zeta_{2}^{2}$.

- Consider now the map $H_{*}(M U ; \mathbb{Z} / 2) \rightarrow x^{*}(\mathcal{V})$; observe that this is a morphism of graded algebras. In $x^{*}(\mathcal{V})$, one has an element $s$ in degree 2, an element $t$ in degree 6 , and one has a basis for the algebra given by $\left\{1, s, s^{2}, s^{3}, t, s t, s^{2} t, s^{3} t\right\}$ (cf. the proof of Proposition 3.4). In fact, as we saw earlier, the algebra is $\mathbb{Z} / 2[s, t] /\left(s^{4}, t^{2}\right)$. Since $H_{*}(M U ; \mathbb{Z} / 2) \rightarrow x^{*}(\mathcal{V})$ is a surjection of graded algebras, this means that the indecomposable element $S$ in degree 2 of $H_{*}(M U ; \mathbb{Z} / 2)$ must map to $s$ in $x^{*}(\mathcal{V})$, and the indecomposable element $T$ in degree 6 must map to $t$ mod decomposables.

Combining these two observations, it now follows that the composite map $x^{*} \mathcal{F}(D A(1)) \rightarrow x^{*} \mathcal{V}$ is an isomorphism, as desired.

\subsection{Calculation of $\operatorname{Tmf}_{*}(D A(1))$}

We now have done the work necessary to compute $\operatorname{Tmf}_{*}(D A(1))$. As before, let $T=\operatorname{Spec}\left(\mathbb{Z}_{(2)}\left[\alpha_{1}, \alpha_{3}\right]\right) / \mathbb{G}_{m}$. Note that it is not $T$ and $M_{c u b}$ which are relevant to this computation, but rather $T \times_{M_{c u b}} M_{\overline{e l l}}$ and $M_{\overline{e l l}}$. We will use the descent spectral sequence (4) and the determination of $\mathcal{F}(D A(1))$ of the previous subsections.

Lemma 4.8. $M_{\overline{\text { ell }}} \times_{M_{\text {cub }}} T \simeq \mathbb{P}(1,3)$ is the weighted projective stack: that is, the complement of the intersection $V\left(\alpha_{1}\right) \cap V\left(\alpha_{3}\right)$ in $\operatorname{Spec}\left(\mathbb{Z}_{(2)}\left[\alpha_{1}, \alpha_{3}\right]\right) / \mathbb{G}_{m}$.

Proof. We recall (cf. [5]) that the substack $M_{\overline{e l l}} \subset M_{c u b}$ is the complement of the closed substack of cuspidal curves cut out by the vanishing of the modular forms $c_{4}, \Delta$. It follows that $M_{\overline{\text { ell }}} \times_{M_{\text {cub }}} T$ is the substack of $T \simeq \operatorname{Spec}\left(\mathbb{Z}_{(2)}\left[\alpha_{1}, \alpha_{3}\right]\right) / \mathbb{G}_{m}$ complementary to that cut out by the vanishing of $c_{4}, \Delta$. In other words, we need to show that $c_{4}, \Delta$ generate an ideal in $\mathbb{Z}_{(2)}\left[\alpha_{1}, \alpha_{3}\right]$ which contains all elements of sufficiently large degree.

We can again show this after reducing mod 2. Here we use the expressions mod 2 for $c_{4}, \Delta$ of the cubic curve $y^{2}+\alpha_{1} x y+\alpha_{3} y=x^{3}$ (easily extracted from [5, p. 57]); they are given by

$$
c_{4} \equiv \alpha_{1}^{4}, \quad \Delta \equiv\left(\alpha_{1} \alpha_{3}\right)^{3}+\alpha_{3}^{4} .
$$

These together imply that $c_{4}, \Delta$ cut out the empty subscheme of $\mathbb{P}_{\mathbb{Z} / 2}^{1}$ and consequently generate a power of the irrelevant ideal.

Theorem 4.9. The descent spectral sequence for $\pi_{*}(\operatorname{Tmf} \wedge D A(1))$ collapses. The terms in nonnegative degrees are given (additively) by $\mathbb{Z}_{(2)}\left[\alpha_{1}, \alpha_{3}\right]$ : that is, $\pi_{*} \tau_{\geqslant 0}(\operatorname{Tmf} \wedge D A(1)) \simeq \mathbb{Z}_{(2)}\left[\alpha_{1}, \alpha_{3}\right]$. Here $\left|\alpha_{1}\right|=2,\left|\alpha_{3}\right|=6$.

Proof. We saw in Proposition 4.7 that if $p: \mathbb{P}(1,3) \rightarrow M_{\overline{\text { ell }}}$ was the eight-fold cover as above, then $\mathcal{F}(D A(1)) \simeq p_{*}(\mathcal{O})$, so that by the projection formula,

$$
\mathcal{F}(D A(1)) \otimes \omega^{j} \simeq p_{*}\left(\omega^{j}\right) .
$$

Here $\omega$ refers to the usual bundle on $M_{F G}$ or $M_{\overline{\text { ell }}}$. Over $\mathbb{P}(1,3)$, it arises simply from a shift in grading, i.e., from the graded $\mathbb{Z}_{(2)}\left[\alpha_{1}, \alpha_{3}\right]$-module $\mathbb{Z}_{(2)}\left[\alpha_{1}, \alpha_{3}\right] \iota$ where $|\iota|=-2$. 
Consequently, we have the $E_{2}$-term of the descent spectral sequence:

$$
H^{i}\left(M_{\overline{e l l}}, \mathcal{F}(D A(1)) \otimes \omega^{j}\right)=H^{i}\left(\mathbb{P}(1,3), \omega^{j}\right) .
$$

The cohomology of a weighted projective stack is the same as the classical cohomology of projective space, but the grading is modified. Namely, one has to compute the cohomology of $\mathbb{A}^{2} \backslash\{(0,0)\}=\operatorname{Spec}\left(\mathbb{Z}_{(2)}\left[\alpha_{1}, \alpha_{3}\right]\right) \backslash V\left(\alpha_{1}, \alpha_{3}\right)$ and keep track of the grading. We find that $H^{\bullet}\left(\mathbb{A}^{2} \backslash\{(0,0)\}, \mathcal{O}\right)$ is the cohomology of the two-term (Cech) complex:

$$
\mathbb{Z}_{(2)}\left[\alpha_{1}^{ \pm 1}, \alpha_{3}\right] \oplus \mathbb{Z}_{(2)}\left[\alpha_{1}, \alpha_{3}^{ \pm 1}\right] \rightarrow \mathbb{Z}_{(2)}\left[\alpha_{1}^{ \pm 1}, \alpha_{3}^{ \pm 1}\right]
$$

and the cohomology of $\mathbb{P}(1,3)$ is the same, with the grading taken into account. In particular, the spectral sequence (8) is concentrated in the bottom two rows, and each row is easy to describe. We have:

$$
\begin{gathered}
H^{0}\left(\mathbb{A}^{2} \backslash\{(0,0)\}, \mathcal{O}\right)=\mathbb{Z}_{(2)}\left[\alpha_{1}, \alpha_{3}\right], \\
H^{1}\left(\mathbb{A}^{2} \backslash\{(0,0)\}, \mathcal{O}\right)=\mathbb{Z}_{(2)}\left\{\alpha_{1}^{-1} \alpha_{3}^{-1}, \alpha_{1}^{-2} \alpha_{3}^{-1}, \alpha_{1}^{-1} \alpha_{3}^{-2}, \ldots\right\} .
\end{gathered}
$$

By the gap theorem in Theorem 2.7, we have

$$
\tau_{\geqslant 0}(\operatorname{Tmf} \wedge D A(1)) \simeq \operatorname{tmf} \wedge D A(1) .
$$

In particular, we also get:

Corollary 4.10. We have an additive isomorphism $\operatorname{tmf}_{*}(D A(1)) \simeq \mathbb{Z}_{(2)}\left[\alpha_{1}, \alpha_{3}\right]$.

\subsection{Connections with level structures}

We start by reviewing the modular interpretation of the eight-fold cover. Over the locus $M_{c u b}\left[\Delta^{-1}\right]$ of (nonsingular) elliptic curves, the restriction of the cover $T \rightarrow M_{c u b}$ is the forgetful functor from the moduli stack of elliptic curves with a $\Gamma_{1}(3)$-structure (i.e., a choice of nonzero 3-torsion point, which here is $(0,0))$ to $M_{c u b}\left[\Delta^{-1}\right]$; see [21]. More generally, over $M_{\overline{e l l}}$, the above cover is the cover $\left(M_{\overline{e l l}}\right)_{1}(3) \rightarrow M_{\overline{e l l}}$ of generalized elliptic curves with a $\Gamma_{1}(3)$-structure [6] (cf. [19, §2]).

The recent work of Hill-Lawson [12] interprets this cover as a morphism of derived stacks. In particular, they construct an even periodic derived version of $\left(M_{\overline{e l l}}\right)_{1}(3)$ which maps to the derived version of the previously constructed $M_{\overline{e l l}}$ (away from the prime 3$)$. The global sections of the structure sheaf are denoted $\operatorname{Tmf}_{1}(3)$ and its connective cover is $\operatorname{tmf}_{1}(3)$.

Let $\mathcal{O}^{\text {top }}$ be the sheaf of $E_{\infty}$-ring spectra on the étale site of $M_{\overline{e l l}}$. Previously, we identified the quasi-coherent sheaf on $M_{\overline{\text { ell }}}$ obtained by taking $\pi_{0}\left(\mathcal{O}^{\text {top }} \wedge D A(1)\right)$, and we showed that it identifies with the pushforward of the structure sheaf along $\left(M_{\overline{e l l}}\right)_{1}(3) \rightarrow M_{\overline{e l l}}$. As a consequence of the Hill-Lawson work, we can upgrade this to an identification of quasi-coherent sheaves on derived stacks.

Corollary 4.11. The following two quasi-coherent sheaves of $\mathcal{O}^{\text {top }}$-modules (on $M_{\overline{-l l}}$ localized at 2) are equivalent:

1. $D A(1) \wedge \mathcal{O}^{\text {top }}$.

2. The pushforward of the structure sheaf $\mathcal{O}^{\text {top }}$ on the derived version of $\left(M_{\overline{e l l}}\right)_{1}(3)$ to $M_{\overline{e l l}}$. 
Proof. Both yield quasi-coherent sheaves $\mathcal{Q}_{1}, \mathcal{Q}_{2}$ of $\mathcal{O}^{\text {top }}$-modules on $M_{\overline{e l l}}$ which are locally free, and Proposition 4.7 shows that the vector bundles on $M_{\overline{e l l}}$ given by taking $\pi_{0}$ are isomorphic. In order to produce an equivalence $\mathcal{Q}_{1} \simeq \mathcal{Q}_{2}$, we need to produce a global section of $\mathcal{Q}_{3}=\mathcal{Q}_{1} \otimes_{\mathcal{O}^{\text {top }}} \operatorname{Hom}_{\mathcal{O}^{\text {top }}}\left(\mathcal{Q}_{2}, \mathcal{O}^{\text {top }}\right)$. Now $\mathcal{Q}_{3}$ is a sheaf of $\mathcal{O}^{\text {top }}$-modules such that on $\pi_{0}$, one obtains the vector bundle $\mathcal{V} \otimes \mathcal{V}^{*}$ on $M_{\overline{e l l}}$, and the unit is a global section of $\pi_{0} \mathcal{Q}_{3}$. This survives to a global section of $\mathcal{Q}_{3}$ because $\pi_{*} \mathcal{Q}_{3}$ has only cohomology in $H^{0}$ and $H^{1}$ as it is pushed forward from $\left(M_{\overline{e l l}}\right)_{1}(3)$, which has cohomological dimension one. In particular, there are no obstructions to producing the equivalence $\mathcal{Q}_{1} \simeq \mathcal{Q}_{2}$.

Taking global sections and connective covers (using the gap in $\pi_{*}$ Tmf), one obtains:

Theorem 4.12 (Hopkins-Mahowald). We have an equivalence of $\operatorname{tmf}_{(2)}$-modules $\operatorname{tmf}_{(2)} \wedge D A(1) \simeq\left(\operatorname{tmf}_{1}(3)\right)_{(2)}$.

\subsection{Analogs at odd primes}

We briefly indicate the modifications in the above arguments that can be used at an odd prime.

When localized at a prime $p>3$, the moduli stack $M_{\overline{e l l}}$ can be identified with the weighted projective stack $\mathbb{P}(4,6)$ : that is, any (possibly nodal) elliptic curve over a $\mathbb{Z}[1 / 6]$-algebra $R$ can be (Zariski locally) written in the form $y^{2}=x^{3}+A x+B$, where $A, B$ do not simultaneously vanish. The isomorphisms between elliptic curves are of the form $(x, y) \mapsto\left(u^{2} x, u^{3} y\right)$ for $u \in R^{*}$. The elements $A$ and $B$ are, up to units in $R$, the modular forms $c_{4}, c_{6}$. In particular, the descent spectral sequence for $\operatorname{Tmf}_{(p)}$ runs

$$
H^{i}\left(\mathbb{P}(4,6), \omega^{j}\right) \Longrightarrow \pi_{2 j-i} \operatorname{Tmf}_{(p)}
$$

and degenerates, since the cohomology is concentrated in $H^{0}$ and $H^{1}$. One has therefore

$$
\pi_{*} \operatorname{Tmf}=\mathbb{Z}_{(p)}\left[c_{4}, c_{6}\right] \oplus \mathbb{Z}_{(p)}\left\{c_{4}^{-1} c_{6}^{-1}, c_{4}^{-2} c_{6}^{-1}, c_{4}^{-1} c_{6}^{-2}, \ldots\right\}
$$

and Tmf is complex-orientable as it is torsion-free.

Next we consider the prime 3 . Here there is a three-fold cover of the moduli stack of cubic curves. We will construct this cover, and show that it can be realized via a three-cell complex.

Proposition 4.13. Let $\bar{p}$ : $\operatorname{Spec}\left(\mathbb{Z}_{(3)}\left[\alpha_{2}, \alpha_{4}\right]\right) / \mathbb{G}_{m} \rightarrow M_{\text {cub }}$ classify the cubic curve $y^{2}=x^{3}+\alpha_{2} x^{2}+\alpha_{4} x$. Then $\bar{p}$ is a finite flat cover of rank three.

Proof. We will imitate the arguments of Proposition 3.4. Namely, to show finiteness, we can argue as in the proof of Proposition 3.4 and reduce to showing that the pullback $\operatorname{Spec}\left(\mathbb{Z}_{(3)}\left[a_{2}, a_{4}\right]\right) \times_{M_{\text {cub }}} \operatorname{Spec}(\mathbb{Z} / 3)$ is the spectrum of a finite $\mathbb{Z} / 3$-algebra (where $\operatorname{Spec}(\mathbb{Z} / 3) \rightarrow M_{\text {cub }}$ classifies the cuspidal cubic). Using the change-of-variable formulas, we find that this fiber product is the spectrum of

$$
\mathbb{Z} / 3[r, s, t] /\left(2 s, 2 t, r^{3}-t^{2}\right)=\mathbb{Z} / 3[r] /\left(r^{3}\right),
$$

which is clearly a finite $\mathbb{Z} / 3$-algebra of dimension three. We conclude that for any $R$ and for any map $\operatorname{Spec}(R) \rightarrow M_{c u b}$, the fiber product $\operatorname{Spec}\left(\mathbb{Z}_{(3)}\left[a_{2}, a_{4}\right]\right) / \mathbb{G}_{m} \times_{M_{c u b}}$ $\operatorname{Spec}(R)$ is a subscheme of $\mathbb{A}_{R}^{3}$ cut out by three equations, and that it is finite over $R$. As in the proof of Proposition 3.4, this implies flatness. 
In order to realize the vector bundle $\bar{p}_{*}(\mathcal{O})$ on $M_{\overline{\text { ell }}}$ by a spectrum, we consider the generating element $\alpha_{1} \in \pi_{3}\left(S^{0}\right)_{(3)}=\mathbb{Z} / 3$. The cofiber of $\alpha_{1}$, which is a desuspension of $\mathbb{H}^{2}$, has cohomology generated by elements $x_{0}, x_{4}$ with $\mathcal{P}^{1} x_{0}=x_{4}$. Since, furthermore, $\alpha_{1}^{2}=0 \in \pi_{6}\left(S^{0}\right)_{(3)}=0$, we conclude that there is a 3 -local finite spectrum $X_{3}$ such that

$$
H^{\bullet}\left(X_{3} ; \mathbb{Z} / 3\right) \simeq\left\{x_{0}, x_{4}, x_{8}\right\}, \quad \mathcal{P}^{1} x_{0}=x_{4}, \mathcal{P}^{1} x_{4}=x_{8} .
$$

To construct $X_{3}$, we consider the diagram

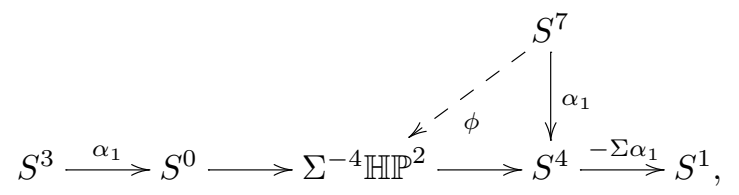

where the horizontal line is a cofiber sequence. Since $\alpha_{1}^{2}=0$, we find a lifting $\phi: S^{7} \rightarrow$ $\Sigma^{-4} \mathbb{H} \mathbb{P}^{2}$ and let $X_{3}$ be the cofiber of $\phi$.

In the spirit of the previous sections, we prove:

Proposition 4.14. The vector bundle that $X_{3}$ defines on $M_{\overline{e l l}}$ is isomorphic to $\bar{p}_{*}(\mathcal{O})$.

Proof. We follow the outline of the earlier arguments. In fact, we start by producing a map $X_{3} \rightarrow M U$ (implicitly localized at 3 ) which induces an isomorphism on $\pi_{0}$, using the degeneration of the AHSS. In homology, the map

$$
H_{*}\left(X_{3} ; \mathbb{Z} / 3\right) \rightarrow H_{*}(M U ; \mathbb{Z} / 3) \simeq \mathbb{Z} / 3\left[x_{1}, x_{2}, \ldots,\right], \quad\left|x_{i}\right|=2 i
$$

is an embedding whose image contains an indecomposable generator in degree four, and its square. As a result, one gets a map of vector bundles on $M_{F G}, \mathcal{F}\left(X_{3}\right) \rightarrow$ $\mathcal{F}(M U)$, such that when one takes the fiber over the additive formal group over $\operatorname{Spec}(\mathbb{Z} / 3)$, one obtains the above map in homology.

Next, consider the cover $q: \operatorname{Spec}(L) / \mathbb{G}_{m} \rightarrow M_{F G}$ and the pullback (cf. Theorem 4.4)

$$
q^{\prime}: \operatorname{Spec}\left(\mathbb{Z}_{(3)}\left[a_{1}, \ldots, a_{6},\left\{e_{n}\right\}_{n \geqslant 4}\right]\right) / \mathbb{G}_{m} \simeq M_{\text {cub }}^{\text {coord }} \rightarrow M_{\text {cub }} .
$$

As before, $q_{*}^{\prime}(\mathcal{O})$ is the sheaf on $M_{c u b}$ that one obtains from $M U$. One produces a map $q_{*}^{\prime}(\mathcal{O}) \rightarrow \bar{p}_{*}(\mathcal{O})$, by considering the closed embedding

$$
\operatorname{Spec}\left(\mathbb{Z}_{(3)}\left[\alpha_{2}, \alpha_{4}\right]\right) / \mathbb{G}_{m} \hookrightarrow \operatorname{Spec}\left(\mathbb{Z}_{(3)}\left[a_{1}, \ldots, a_{6},\left\{e_{n}\right\}_{n \geqslant 4}\right]\right) / \mathbb{G}_{m},
$$

which sends $a_{2} \mapsto \alpha_{2}, a_{4} \mapsto \alpha_{4}$, and annihilates all the other generators.

The claim is that the composite map $\mathcal{F}\left(X_{3}\right) \rightarrow \mathcal{F}(M U) \rightarrow \bar{p}_{*}(\mathcal{O})$, is an isomorphism of vector bundles on $M_{c u b}$, which as before can be checked by showing that the map yields a surjection when one takes the fiber over the cuspidal cubic. To see this, we observe that when one takes the fiber over the cuspidal cubic, one gets the embedding $H_{*}\left(X_{3} ; \mathbb{Z} / 3\right) \rightarrow H_{*}(M U ; \mathbb{Z} / 3)$, whose image contains an indecomposable generator in degree 4 and its square. The map from $H_{*}(M U ; \mathbb{Z} / 3)$ to the fiber of $\bar{p}_{*}(\mathcal{O})$ over the cuspidal cubic (which we have checked to be $\mathbb{Z} / 3[r] /\left(r^{3}\right)$ ) is a map of graded algebras and induces a surjection on indecomposables. From this, the conclusion follows similarly. 
The threefold cover of $M_{\overline{e l l}}$ one obtains here is obtained from $\Gamma_{1}(2)$-structures [31, $\S 7]$. Using similar techniques as in Theorem 4.12 and the Hill-Lawson work [12], one obtains from the above analysis:

Theorem 4.15. One has an equivalence of $\operatorname{tmf}_{(3)}$-modules $\operatorname{tmf}_{(3)} \wedge X_{3} \simeq \operatorname{tmf}_{1}(2)_{(3)}$.

\section{The remaining computations}

In this section, we finish the computations. We use the 2-local complex $D A(1)$ and the 3-local complex $F$ to analyze the Adams-Novikov spectral sequence for tmf. We then identify the spectrum $\operatorname{tmf}_{(2)} \wedge D A(1) \simeq \operatorname{tmf}_{1}(3)_{(2)}$ with a form of $B P\langle 2\rangle$, and using Hopf algebra manipulations, we are able to recover $H_{*}(\operatorname{tmf} ; \mathbb{Z} / 2)$.

\subsection{Calculation of $\operatorname{Tmf}_{*}(M U)$ and the stack for tmf}

In this subsection, we compute the homotopy groups of $\operatorname{Tmf} \wedge M U$ and $\operatorname{tmf} \wedge M U$. This leads to the description of the Adams-Novikov spectral sequence for tmf in terms of the moduli stack of cubic curves. Throughout, we work integrally.

Proposition 5.1. Let $R=\mathbb{Z}\left[a_{1}, \ldots, a_{6},\left\{e_{n}\right\}\right]_{n \geqslant 4}$. Then $\operatorname{Tmf}_{*}(M U)$ is a module over $R$. As an $R$-module, it is isomorphic to $R \oplus C$, where $C_{*}=\left(R /\left(c_{4}^{\infty}, \Delta^{\infty}\right)\right)_{*+1}$ and $R /\left(c_{4}^{\infty}, \Delta^{\infty}\right)$ is the cokernel of $R\left[c_{4}^{-1}\right] \oplus R\left[\Delta^{-1}\right] \rightarrow R\left[\left(c_{4} \Delta\right)^{-1}\right]$.

Proof. This follows from the descent spectral sequence $H^{i}\left(M_{\overline{e l l}}, \mathcal{F}(M U) \otimes \omega^{j}\right) \Longrightarrow$ $\pi_{2 j-i}(\operatorname{Tmf} \wedge M U)$. The sheaf $\mathcal{F}(M U)$ on $M_{\overline{e l l}}$ is obtained by pushing forward the structure sheaf along the affine map

$$
\operatorname{Spec}\left(\pi_{*} M U\right) / \mathbb{G}_{m} \times_{M_{F G}} M_{\overline{e l l}} \rightarrow M_{\overline{e l l}} .
$$

The source of this morphism is identified with $\left(\operatorname{Spec}(R) \backslash V\left(c_{4}, \Delta\right)\right) / \mathbb{G}_{m}$ by Theorem 4.4, since the locus $M_{\overline{e l l}} \subset M_{c u b}$ is the complement of the closed substack defined by $c_{4}, \Delta$.

Let $B$ be the scheme $\operatorname{Spec}(R) \backslash V\left(c_{4}, \Delta\right)$. Consequently, we get for the $E_{2}$ page of the descent spectral sequence for $\operatorname{Tmf}_{*}(M U)$,

$$
E_{2}^{i, 2 j}=H^{i}\left(M_{\overline{e l l}}, \mathcal{F}(M U) \otimes \omega^{j}\right) \simeq H^{i}(B, \mathcal{O})_{j},
$$

where the subscript $j$ denotes taking the $j$ th piece. The scheme $B$ is covered by two affine opens given by the localizations at $\Delta$ and $c_{4}$. In particular, it has cohomological dimension one, and for dimensional reasons the descent spectral sequence for $\operatorname{Tmf} \wedge$ $M U$ degenerates. We can describe the cohomology of $B$ as that of the Cech complex

$$
R\left[c_{4}^{-1}\right] \oplus R\left[\Delta^{-1}\right] \rightarrow R\left[\left(c_{4} \Delta\right)^{-1}\right] .
$$

Putting this together, the result follows easily as the $H^{0}$ gives $R$ and the $H^{1}$ gives $R /\left(c_{4}^{\infty}, \Delta^{\infty}\right)$.

We observe that $C$ can be identified with the ideal of $\operatorname{Tmf}_{*}(M U)$ given by elements in positive filtration degree in the filtration from the descent spectral sequence, and consequently $C^{2}=0$.

We will now describe the stack corresponding to tmf, or equivalently describe the structure of the Adams-Novikov spectral sequence for tmf. We will see that the stack 
associated to tmf (in the sense of sec. 2.2) is precisely the moduli stack $M_{c u b}$ of cubic curves. This produces a spectral sequence that allows computation with tmf.

Corollary 5.2 (Cf. [29, Prop. 20.1]). One has

$$
\pi_{*}(\operatorname{tmf} \wedge M U)=\mathbb{Z}\left[a_{1}, a_{2}, a_{3}, a_{4}, a_{6},\left\{e_{n}\right\}\right]_{n \geqslant 4} .
$$

Proof. We begin by computing $\operatorname{tmf}_{*}(M U)$. In fact, we know (Proposition 5.1) that $\pi_{*}(\operatorname{Tmf} \wedge M U)$ canonically surjects onto the ring in question with kernel an ideal $C \subset \operatorname{Tmf}_{*}(M U)$ of square zero, and that there is a map $\operatorname{tmf} \wedge M U \rightarrow \operatorname{Tmf} \wedge M U$. The claim is that the composite

$$
\operatorname{tmf}_{*}(M U) \rightarrow \operatorname{Tmf}_{*}(M U) \rightarrow \operatorname{Tmf}_{*}(M U) / C \simeq \operatorname{Tmf}_{*}(M U) / F_{1} \operatorname{Tmf}_{*}(M U)
$$

is an isomorphism, where we use the notation of Proposition 5.1 for $C$ and where $F_{1}$ refers to the filtration from the descent spectral sequence. This will compute $\operatorname{tmf}_{*}(M U)$, as desired. We will prove this locally at each prime $p$.

In order to prove this, we will use the existence of a finite even $p$-local spectrum $Z$ with the following properties:

1. $\operatorname{Tmf} \wedge Z$ is a complex-orientable ring spectrum.

2. $\operatorname{tmf} \wedge Z=\tau_{\geqslant 0}(\operatorname{Tmf} \wedge Z)$.

For $p=2$, we proved this fact with $Z=D A(1)$. For $p=3$, we proved this fact with $Z=X_{3}$. For $p \geqslant 5$, we can take $Z=S^{0}$, since Tmf and tmf are complex-orientable ring spectra with torsion-free homotopy groups.

We will now use these three facts to prove that (9) is an isomorphism at the arbitrary prime $p$. To start with, we note that $\operatorname{tmf} \wedge M U$ is a complex-orientable $E_{\infty}$-ring, so that, since $Z$ is an even spectrum, $\operatorname{tmf}_{*}(M U \wedge Z)$ is a sum of copies of $\operatorname{tmf}_{*}(M U)$ (possibly shifted). The analog holds for $\operatorname{Tmf}_{*}(M U \wedge Z)$. Moreover, since the map tmf $\wedge Z \rightarrow \operatorname{Tmf} \wedge Z$, induces a split injection on homotopy groups, we find that

$$
\pi_{*}(\operatorname{tmf} \wedge Z \wedge M U) \rightarrow \pi_{*}(\operatorname{Tmf} \wedge Z \wedge M U)
$$

is a split injection of graded abelian groups in view of the (known) $M U$-homology of any complex-oriented ring spectrum.

Since $Z$ is even, we conclude that $\operatorname{tmf}_{*}(M U) \rightarrow \operatorname{Tmf}_{*}(M U)$ is a split injection. It follows that both $\operatorname{tmf}_{*}(M U)$ and the cokernel of $\operatorname{tmf}_{*}(M U) \rightarrow \operatorname{Tmf}_{*}(M U)$ are torsion-free abelian groups. To show that $\operatorname{tmf}_{*}(M U) \cap F_{1} \operatorname{Tmf}_{*}(M U)=0$ and that $\operatorname{tmf}_{*}(M U) \rightarrow \operatorname{Tmf}_{*}(M U) / F_{1} \operatorname{Tmf}_{*}(M U)$ is an isomorphism, it now suffices to work rationally, since the kernel and the cokernel are torsion-free. This is much easier. In fact, rationally, the descent spectral sequence for Tmf degenerates and is concentrated in the zeroth and first row. The image of $\pi_{*} \operatorname{tmf} \otimes \mathbb{Q} \rightarrow \pi_{*} \operatorname{Tmf} \otimes \mathbb{Q}$ consists of the elements on the zeroth row: that is, $\pi_{*} \operatorname{tmf} \otimes \mathbb{Q} \rightarrow \pi_{*} \operatorname{Tmf} \otimes \mathbb{Q} \rightarrow\left(\pi_{*} \operatorname{Tmf} / F_{1} \pi_{*} \operatorname{Tmf}\right) \otimes$ $\mathbb{Q}$ is an isomorphism. It follows that this is true after tensoring tmf with any rational spectrum.

In particular, we can describe the spectral sequence for tmf-homology.

Corollary 5.3 (Hopkins-Mahowald). The stack for tmf is identified with $M_{\text {cub }}$. In particular, let $X$ be a spectrum. Then $X$ defines a quasi-coherent sheaf $\widetilde{F}(X)$ on the 
moduli stack $M_{\text {cub }}$ (if $X$ is even, then $\widetilde{F}(X)$ is the pullback of $\mathcal{F}(X)$ under $M_{\text {cub }} \rightarrow$ $\left.M_{F G}\right)$ and a spectral sequence

$$
H^{i}\left(M_{c u b}, \widetilde{F}(X) \otimes \omega^{j}\right) \Longrightarrow \operatorname{tmf}_{2 j-i}(X) .
$$

In [29], Rezk uses instead the Thom spectrum $X(4)$ and reproduces the Weierstrass Hopf algebroid (cf. [29, Prop. 12.4 and Th. 14.5]).

Proof. This analysis shows that the morphism $\operatorname{tmf}_{*}(M U) \rightarrow \operatorname{Tmf}_{*}(M U) \rightarrow$ $\operatorname{Tmf}_{*}(M U) / C$ is an isomorphism, where $C$ is the ideal consisting of those elements of filtration 1 in the (degenerate) descent spectral sequence for $\operatorname{Tmf} \wedge M U$. The same is therefore true when $M U$ is replaced by any wedge of suspensions of $M U$, for instance any smash power of $M U$. We find that, for any $s>0, \operatorname{tmf}_{*}\left(M U^{\wedge s}\right)$ can be described as global sections of the structure sheaf on the stack

$$
M_{\text {ell }}^{\text {coord }} \times_{M_{\overline{e l l}}} \cdots \times \times_{M_{\overline{e l l}}} M_{\text {ell }}^{\text {coord }}
$$

(with $s$ factors): that is, the contributions of $H^{1}$ terms in the Tmf-homology can be ignored. The ring of global sections over this stack is the same as the ring of global sections over the larger stack

$$
M_{c u b}^{\text {coord }} \times_{M_{\text {cub }}} \cdots \times_{M_{\text {cub }}} M_{\text {cub }}^{\text {coord }},
$$

which differs by a substack of codimension 2. We find that the Hopf algebroid

$$
\operatorname{tmf}_{*}(M U) \stackrel{\rightarrow}{\rightarrow} \operatorname{tmf}_{*}(M U \wedge M U) \stackrel{\rightarrow}{\rightarrow} \cdots
$$

precisely writes down the presentation of $M_{c u b}$ via the flat cover $M_{c u b}^{\text {coord }} \rightarrow M_{c u b}$.

The stack $M_{c u b}$ can be presented by the Weierstrass Hopf algebroid. We remark that this spectral sequence is the Adams-Novikov spectral sequence for $\operatorname{tmf} \wedge X$. In [4], it is used to calculate $\pi_{*}$ tmf.

\section{2. $\operatorname{tmf} \wedge D A(1)$}

We will now identify the ring spectrum $\operatorname{tmf}_{1}(3)$ with a form of $B P\langle 2\rangle$.

We begin with some generalities. Implicitly, we work at a prime $p$. Given an $E_{\infty}$ ring spectrum $R$ and an element $r \in \pi_{k}(R)$, we write $R / r$ for the cofiber of $r: \Sigma^{k} R \rightarrow$ $R$. Given a sequence of elements $r_{1}, r_{2}, \ldots, \in \pi_{*}(R)$, we let $R /\left(r_{1}, r_{2}, \ldots\right)$ denote the colimit over $n$ of the finite smash products $R / r_{1} \wedge_{R} \cdots \wedge_{R} R / r_{n}$. When $\pi_{*}(R)$ is concentrated in even degrees and the $r_{i}$ 's are nonzerodivisors in $\pi_{*}(R)$, it is known that these can always be given $A_{\infty}$-algebra structures in $R$-modules (cf. [2, Sec. 3]).

Definition 5.4. A $M U$-module spectrum $M$ is said to be a form of $B P\langle n\rangle$ (at the prime $p$ ) if there exist elements $x_{i} \in \pi_{2 i} M U$ for $i \in \mathbb{Z}_{>0} \backslash\left\{p-1, p^{2}-1, \ldots, p^{n}-1\right\}$ such that one has an equivalence of $M U$-modules

$$
M U_{(p)} /\left(\left\{x_{i}\right\}\right) \simeq M
$$

and such that $x_{i}$ generates $\pi_{2 i}(M U)$ modulo decomposables. We will frequently abuse notation and write $B P\langle n\rangle$ for any form of $B P\langle n\rangle$.

In general, it does not seem easy to tell whether an $M U$-module spectrum is a form of $B P\langle n\rangle$ simply from looking at its homotopy groups as a module over $M U_{*}=$ 
$\pi_{*}(M U)$. However, the following examples will be useful in the future. We note also that recent work of Angeltveit-Lind [3] has shown that the spectrum obtained by $p$-completing $B P\langle n\rangle$ is determined by its $\bmod p$ cohomology.

Example 5.5. Suppose $M$ is an $M U$-module spectrum with

$$
M_{*} \simeq B P_{*} /\left(v_{n+1}, v_{n+2}, \ldots,\right) \simeq \mathbb{Z}_{(p)}\left[v_{1}, v_{2}, \ldots, v_{n}\right] .
$$

Suppose $M$ admits the structure of an $M U$-ring spectrum, i.e., the structure of a possibly nonassociative algebra object in the homotopy category of $M U$-modules, inducing the natural ring structure on $M_{*}$. Then $M$ is a form of $B P\langle n\rangle$.

In fact, consider the map $M U_{*} \rightarrow M_{*}$ induced by the unit $M U \rightarrow M$, and choose indecomposable generators $y_{i}$ in $\pi_{2 i}(M U)$ (for each $i \neq p-1, p^{2}-1, \ldots, p^{n}-1$ ) for the kernel. The unit map $M U \rightarrow M$ extends over $M U / y_{i} \rightarrow M$, and we can take the smash product of all of these together and localize at $p$ to get a map $M U_{(p)} /\left(\left\{y_{i}\right\}\right) \simeq$ $B P\langle n\rangle \rightarrow M$, which is an isomorphism on homotopy groups.

Observe that $\operatorname{tmf} \wedge M U$ is an $E_{\infty}$-ring spectrum, so that we can make various quotients in the category of $\operatorname{tmf} \wedge M U$-modules. Consider in particular $\left(\operatorname{tmf}_{(2)} \wedge\right.$ $M U) /\left(a_{2}, a_{4}, a_{6},\left\{e_{n}\right\}\right)$. The next lemma will enable us to analyze it.

Lemma 5.6. The homotopy groups of the $M U$-ring spectrum $\operatorname{tmf}(2) \wedge M U /\left(a_{2}, a_{4}\right.$, $\left.a_{6},\left\{e_{n}\right\}\right)$ are given by $\mathbb{Z}_{(2)}\left[a_{1}, a_{3}\right]$, and it is a form of $B P\langle 2\rangle$.

Let $I$ denote the ideal $\left(a_{2}, a_{4}, a_{6},\left\{e_{n}\right\}\right) \subset R$; we will (by abuse of notation) write $\left(\operatorname{tmf}_{(2)} \wedge M U\right) / I$ for the quotient $\left(\operatorname{tmf}_{(2)} \wedge M U\right) /\left(a_{2}, a_{4}, a_{6},\left\{e_{n}\right\}\right)$.

Proof. It suffices to describe the Hurewicz map $M U_{*} \rightarrow M_{*}$, in view of Example 5.5. By [2, Cor. 3.2], $M$ is an $A_{\infty}$-algebra in $M U$-modules.

Given an elliptic spectrum $E$ associated to an elliptic curve over $\operatorname{Spec}(R)$, the map $M U_{*} \rightarrow E_{*}(M U)$ yields the map $\operatorname{Spec}\left(E_{*}(M U)\right) \rightarrow \operatorname{Spec}\left(M U_{*}\right)$ from the ring classifying coordinates on the formal group to the ring classifying formal group laws. In particular, the map

$$
M U_{*} \rightarrow \operatorname{tmf}_{*}(M U) \simeq \operatorname{Tmf}_{*}(M U) / F_{1} \operatorname{Tmf}_{*}(M U)
$$

classifies the formal group law constructed by choosing the coordinate $-y / x+$ $\sum_{n \geqslant 4} e_{n}(-y / x)^{n+1}$ on the formal group of $y^{2}+a_{1} x y+a_{3} y=x^{3}+a_{2} x^{2}+a_{4} x+a_{6}$. The composite $M U_{*} \rightarrow\left(\operatorname{Tmf}_{*}(M U) / F_{1} \operatorname{Tmf}_{*}(M U)\right) / I$ classifies the formal group law associated to the coordinate $-y / x$ on $y^{2}+a_{1} x y+a_{3} y=x^{3}$.

Here we use the formulas given in [30, Ex. 4.5, Ch. 4]. The expansion of the power series $[2](z)$ for the formal group law associated to a Weierstrass equation is

$$
[2](z)=2 z-a_{1} z^{2}-2 a_{2} z^{3}+\left(a_{1} a_{2}-7 a_{3}\right) z^{4}+\ldots,
$$

where in our case $a_{2}=0$ and 7 is invertible. In particular, if we take $v_{1}, v_{2}$ to be indecomposable elements of $M U$ in degrees 2 and 6 (e.g., the coefficients of $z^{2}, z^{4}$ in $[2](z))$, and take our equation to be $y^{2}+a_{1} x y+a_{3} y=x^{3}$, then $v_{1}$ maps to a unit times $a_{1}$, and $v_{2}$ maps to a unit times $a_{3}$. It follows that there exist indecomposable generators in $\pi_{i}(M U)$ for $i \neq 2,6$ which generate the kernel of the surjective map $\pi_{*}(M U) \rightarrow \mathbb{Z}_{(2)}\left[a_{1}, a_{3}\right]$. This proves the result. 
Corollary 5.7. The map of $\operatorname{tmf}-$ modules $\operatorname{tmf} \wedge D A(1) \rightarrow(\operatorname{tmf} \wedge M U) / I$ is an equivalence.

Proof. This follows from Proposition 4.7 and from the construction of the vector bundle $\mathcal{V}$ used there. Note that $\pi_{*}(\operatorname{tmf} \wedge D A(1))$ is isomorphic to the quotient of $\pi_{*}(\operatorname{Tmf} \wedge D A(1))$ by the elements in filtration 1 .

Combining Lemma 5.6 and Corollary 5.7, we find:

Theorem 5.8 (Hopkins-Mahowald [14]).

$$
\operatorname{tmf}_{(2)} \wedge D A(1) \simeq \operatorname{tmf}_{1}(3)_{(2)} \simeq \tau_{\geqslant 0}\left(\operatorname{Tmf}_{(2)} \wedge D A(1)\right)
$$

is a form of $B P\langle 2\rangle$.

\subsection{The mod 2 homology of tmf}

In the previous subsection, we established the 2-local equivalence tmf $\wedge D A(1) \simeq$ $B P\langle 2\rangle$. Using Hopf algebra manipulations and the homology of $B P\langle 2\rangle$, we can now calculate the homology of tmf.

We begin by recalling the homology of $B P\langle n\rangle$. The result is classical and follows from the spectral sequence

$$
\operatorname{Tor}_{\pi_{*} M U}\left(\pi_{*} B P\langle n\rangle ; H_{*}(M U ; \mathbb{Z} / 2)\right) \Longrightarrow H_{*}(B P\langle n\rangle ; \mathbb{Z} / 2) ;
$$

see also [19, Th. 4.4].

Proposition 5.9. The map $B P\langle n-1\rangle \rightarrow H \mathbb{Z} / 2$ induces an injection on homology, with image $\mathbb{Z} / 2\left[\zeta_{1}^{2}, \zeta_{2}^{2}, \ldots, \zeta_{n}^{2}, \zeta_{n+1}, \zeta_{n+2}, \ldots\right] \subset \mathcal{A}_{*}$.

We will identify the subalgebra of $H_{*}(B P\langle 2\rangle ; \mathbb{Z} / 2)$ given by the homology of tmf. First, we need a few lemmas. Let $k$ be a field. As usual, a nonnegatively graded $k$ algebra $R$ is called connected if $k \rightarrow R_{0}$ is an isomorphism, and a graded $R$-module $M$ is called connective if it is zero in negative degrees. Given $R$, we let $\bar{R}=\bigoplus_{i>0} R_{i}$. We recall the following:

Lemma 5.10 (Nakayama's lemma). Let $R$ be a graded, connected $k$-algebra with augmentation ideal $\bar{R}$. If $M$ is a connective $R$-module which is flat, then it is free (in particular, if in addition $M \neq 0$, then $M$ is faithfully flat).

Lemma 5.11. Let $B$ be a commutative, graded connected Hopf algebra over a field $k$ and let $A \subset B$ be a graded comodule subalgebra. Then we can write the inclusion $A \subset B$ as a filtered colimit of inclusions $A_{j} \subset B_{j}, j \in J$, where $B_{j} \subset B$ is a finitely generated commutative graded, connected Hopf subalgebra, and $A_{j} \subset B_{j}$ are finitely generated subalgebras which are also comodules for $B_{j}$.

By "commutative graded," we do not mean "graded-commutative." The lemma is false without the graded and connected hypotheses: for instance, consider the ring of functions on the multiplicative group, $k\left[t, t^{-1}\right]$ (a Hopf algebra with $\Delta(t)=t \otimes t$ ), and the comodule subalgebra $k[t]$. Geometrically, this corresponds to the $\mathbb{G}_{m}$-variety $\mathbb{A}^{1}$ : the natural inclusion map $\mathbb{G}_{m} \rightarrow \mathbb{A}^{1}$ (of $\mathbb{G}_{m}$-varieties) is not faithfully flat. We remark that the existence of such structure theorems for comodule algebras in the graded, connected case goes back to [25]. 
Proof. First, observe that $B$ is a filtered colimit of finitely generated graded Hopf subalgebras [23, Lemma 21.1.2]. Observe also that $A$, as a $B$-comodule, is a filtered colimit of finite-dimensional graded $B$-comodules. Given a finite-dimensional $B$-comodule $M \subset A$, it is, as a result, a $\widetilde{B}$-comodule for $\widetilde{B}$ a large enough finitely generated (graded) subHopf algebra of $B$. Now, consider the pairs $(\widetilde{A}, \widetilde{B})$ for $\widetilde{A}$ the subalgebra of $A$ generated by $M$. Then $\widetilde{A}$ is a finitely generated algebra and a $\widetilde{B}$ comodule. Clearly, given any finite collection of elements of $A$ and any finite collection of elements of $B$, we can find a pair $(\widetilde{A}, \widetilde{B})$ containing all of them.

Proposition 5.12. Let $B$ be a commutative graded, connected Hopf algebra over a field $k$, and let $A \subset A^{\prime} \subset B$ be comodule subalgebras. Suppose $B$ is a domain. Then $A^{\prime}$ is graded free over $A$.

Proof. We will show that $B$ is faithfully flat over $A$. This also implies that $B$ is faithfully flat over $A^{\prime}$, and combining these observations shows that $A^{\prime}$ is faithfully flat over $A$. Lemma 5.10 will then imply that $A^{\prime}$ is a free $A$-module. In other words, we may assume $A^{\prime}=B$.

In the notation of Lemma 5.11, we will prove that each of the inclusions $A_{j} \subset B_{j}$ is faithfully flat, which will suffice; in fact we only need to check flatness by Lemma 5.10 again. Thus, we may assume $A$ and $B$ are finitely generated. By making a base change, we may also assume that $k$ is algebraically closed. Then $\operatorname{Spec} B$ is an affine group scheme $G$, of finite type over $k$, and $X=\operatorname{Spec} A$ is a scheme acted on by $G$. There is a map of $G$-schemes $p: G \rightarrow X$ which is dominant (since $A \subset B$ ). Since $X$ is an integral scheme, generic flatness (see, e.g., [8, Theorem 14.4]) implies that there exists a nonempty open subset $U \subset X$ such that $p^{-1}(U) \rightarrow U \rightarrow X$ is flat. Thus for any $g \in G(k)$, we have that $g p^{-1}(U) \rightarrow X$ is flat, and since $G$ is the union of the $g p^{-1}(U)$ for $g \in G(k)$, we find that $G \rightarrow X$ is flat.

Using the above technical tools, we can now deduce our main result.

Theorem 5.13 (Hopkins-Mahowald [14]). The map $\mathrm{tmf} \rightarrow H \mathbb{Z} / 2$ induces an injection on mod 2 homology, and we have an identification

$$
H_{*}(\mathrm{tmf} ; \mathbb{Z} / 2)=\mathbb{Z} / 2\left[\zeta_{1}^{8}, \zeta_{2}^{4}, \zeta_{3}^{2}, \zeta_{4}, \zeta_{5}, \ldots\right] \subset \mathcal{A}_{*} .
$$

Proof. In fact, we know that

$$
H_{*}(B P\langle 2\rangle ; \mathbb{Z} / 2) \simeq \mathbb{Z} / 2\left[\zeta_{1}^{2}, \zeta_{2}^{2}, \zeta_{3}^{2}, \zeta_{4}, \zeta_{5}, \ldots\right],
$$

by Proposition 5.9; the map comes from the truncation $B P\langle 2\rangle \rightarrow H \mathbb{Z}_{(2)} \rightarrow H \mathbb{Z} / 2$ which embeds $H_{*}(B P\langle 2\rangle ; \mathbb{Z} / 2)$ as a subcomodule of $\mathcal{A}_{*}$. We also know that $H_{*}(\operatorname{tmf} ; \mathbb{Z} / 2)$ is a comodule algebra, and the factorization

$$
\operatorname{tmf} \rightarrow \operatorname{tmf} \wedge D A(1) \simeq B P\langle 2\rangle \rightarrow H \mathbb{Z} / 2
$$

shows that it is a subcomodule algebra of $H_{*}(B P\langle 2\rangle ; \mathbb{Z} / 2) \subset \mathcal{A}_{*}$. Moreover, the Künneth formula shows that the graded dimension of $H_{*}(\operatorname{tmf} ; \mathbb{Z} / 2)$ is that of $\mathbb{Z} / 2\left[\zeta_{1}^{8}, \zeta_{2}^{4}, \zeta_{3}^{2}, \zeta_{4}, \ldots\right]$

We will show that if $\mathcal{C}$ is any subcomodule algebra of $H_{*}(B P\langle 2\rangle ; \mathbb{Z} / 2)$ with the same graded dimension as $\mathbb{Z} / 2\left[\zeta_{1}^{8}, \zeta_{2}^{4}, \zeta_{3}^{2}, \zeta_{4}, \ldots\right]$, then $\mathcal{C}$ is in fact $\mathbb{Z} / 2\left[\zeta_{1}^{8}, \zeta_{2}^{4}, \zeta_{3}^{2}, \zeta_{4}, \ldots\right]$ (which is easily checked to be a valid subcomodule algebra). Here we will show that 
any two $\mathcal{C}, \mathcal{C}^{\prime}$ satisfying that condition are equal. In fact, if $\mathcal{C}, \mathcal{C}^{\prime}$ satisfy the condition, then Proposition 5.12 shows that $H_{*}(B P\langle 2\rangle ; \mathbb{Z} / 2)$ is free (necessarily of rank eight) over each of $\mathcal{C}, \mathcal{C}^{\prime}$. Consider the subcomodule algebra $\mathcal{C}^{\prime \prime} \subset H_{*}(B P\langle 2\rangle ; \mathbb{Z} / 2)$ generated by $\mathcal{C}, \mathcal{C}^{\prime}$. It also has the property that $H_{*}(B P ; \mathbb{Z} / 2)$ is free over $\mathcal{C}^{\prime \prime}$, by Proposition 5.12 again. By counting the rank, we will arrive at a contradiction. We need first:

Lemma 5.14. The only elements of $\mathcal{C}^{\prime \prime}$ in degrees $<12$ are 1 and $\xi_{1}^{8}=\zeta_{1}^{8}$.

Proof. In fact, we know that $\operatorname{dim} \mathcal{C}_{8}=\operatorname{dim} \mathcal{C}_{8}^{\prime}=1$, and since this is the smallest dimension of a nonzero element in each of $\mathcal{C}, \mathcal{C}^{\prime}$, the generating element must be primitive. The primitive elements in the dual Steenrod algebra $\mathcal{A}_{*}$, considered as a comodule over itself, are 1 and $\left\{\xi_{1}^{n}\right\}_{n \geqslant 0}$, though, so $\mathcal{C}_{8}=\mathcal{C}_{8}^{\prime}$ is generated by $\xi_{1}^{8}$. There are no other elements in degrees $<12$ in $\mathcal{C}$ or $\mathcal{C}^{\prime}$.

We claim that the eight elements $1, \xi_{1}^{2}, \xi_{1}^{4}, \xi_{1}^{6}, \zeta_{2}^{2}, \xi_{1}^{2} \zeta_{2}^{2}, \xi_{1}^{4} \zeta_{2}^{2}, \xi_{1}^{6} \zeta_{2}^{2} \in H_{*}(B P\langle 2\rangle ; \mathbb{Z} / 2)$ are linearly independent in $H_{*}(B P\langle 2\rangle ; \mathbb{Z} / 2) \otimes_{\mathcal{C}^{\prime \prime}} \mathbb{Z} / 2$ (i.e., could be taken as a subset of generators over $\left.\mathcal{C}^{\prime \prime}\right)$. This is a consequence of the fact that the only elements in $\mathcal{C}^{\prime \prime}$ of degree less than 12 are $1, \xi_{1}^{8}$. Moreover, in degree 12 , we observe that $\xi_{1}^{6} \zeta_{2}^{2} \notin \mathcal{C}^{\prime \prime}$ as it is not primitive modulo $\xi_{1}^{8}$. Consequently, the rank of $H_{*}(B P\langle 2\rangle ; \mathbb{Z} / 2)$ as a $\mathcal{C}^{\prime \prime}$-module must be at least eight. This means that the graded dimension of $\mathcal{C}^{\prime \prime}$ must be equal to that of $\mathcal{C}$, so $\mathcal{C}=\mathcal{C}^{\prime}=\mathcal{C}^{\prime \prime}$.

The dual assertion describes the cohomology via

$$
H^{*}(\operatorname{tmf} ; \mathbb{Z} / 2) \simeq \mathcal{A} \otimes_{\mathcal{A}(2)} \mathbb{Z} / 2,
$$

where $\mathcal{A}(2) \subset \mathcal{A}$ is the subalgebra of the Steenrod algebra $\mathcal{A}$ generated by $\mathrm{Sq}^{1}, \mathrm{Sq}^{2}$, $\mathrm{Sq}^{4}$. In fact, $H^{*}(\mathrm{tmf} ; \mathbb{Z} / 2)$ is cyclic over $\mathcal{A}$ since $H_{*}(\mathrm{tmf} ; \mathbb{Z} / 2) \subset \mathcal{A}_{*}$. Dimensional restrictions force $\mathrm{Sq}^{1}, \mathrm{Sq}^{2}$, and $\mathrm{Sq}^{4}$ to annihilate the generator in degree zero, and this produces a surjection

$$
\mathcal{A} \otimes_{\mathcal{A}(2)} \mathbb{Z} / 2 \rightarrow H^{*}(\mathrm{tmf} ; \mathbb{Z} / 2)
$$

Since $\mathcal{A}(2) \subset \mathcal{A}$ is a $\operatorname{Hopf}$ subalgebra, $\mathcal{A}$ is free over $\mathcal{A}(2)$ by the results of [25], and we find that the graded dimensions of $\mathcal{A} \otimes_{\mathcal{A}(2)} \mathbb{Z} / 2$ and $H^{*}(\operatorname{tmf} ; \mathbb{Z} / 2)$ match. This proves the asserted description of the cohomology.

By the change-of-rings theorem $\operatorname{Ext}_{\mathcal{A}}^{s, t}\left(\mathcal{A} \otimes_{\mathcal{A}(2)} \mathbb{Z} / 2, \mathbb{Z} / 2\right) \simeq \operatorname{Ext}_{\mathcal{A}(2)}^{s, t}(\mathbb{Z} / 2, \mathbb{Z} / 2)$, we now conclude:

Corollary 5.15. The (mod 2) Adams spectral sequence for tmf runs

$$
\operatorname{Ext}_{\mathcal{A}(2)}^{s, t}(\mathbb{Z} / 2, \mathbb{Z} / 2) \Longrightarrow \pi_{t-s} \operatorname{tmf} \otimes \mathbb{Z}_{2}
$$

The Adams spectral sequence for tmf is displayed in [7, Ch. 13]. We remark that (as is well-known) it is not possible to continue this process, and realize modules of the form $\mathcal{A} \otimes_{\mathcal{A}(n)} \mathbb{Z} / 2$ for $n \geqslant 3$, where $\mathcal{A}(n) \subset \mathcal{A}$ is generated by $\left\{\mathrm{Sq}^{1}, \ldots, \mathrm{Sq}^{2^{n}}\right\}$, because of the solution to the Hopf invariant one problem.

This method of computing the homology (and Adams-Novikov spectral sequence) of tmf is dependent on a key piece of prior computational knowledge about Tmf: namely, the gap theorem in the homotopy groups $\pi_{*}$ Tmf. It would be interesting if one could give a theoretical explanation of the gap theorem. 


\section{References}

[1] M. Ando, M.J. Hopkins, and N.P. Strickland. Elliptic spectra, the Witten genus and the theorem of the cube. Invent. Math., 146(3):595-687, 2001.

[2] V. Angeltveit. Topological Hochschild homology and cohomology of $A_{\infty}$ ring spectra. Geom. Topol., 12(2):987-1032, 2008.

[3] V. Angeltveit and J. Lind. Uniqueness of $B P\langle n\rangle$. J. Homotopy Relat. Struct., to appear. DOI 10.1007/s40062-015-0120-0.

[4] T. Bauer. Computation of the homotopy of the spectrum tmf. In Groups, Homotopy and Configuration Spaces, volume 13 of Geom. Topol. Monogr., pages 11-40. Geom. Topol. Publ., Coventry, 2008.

[5] P. Deligne. Courbes elliptiques: formulaire d'après J. Tate. In Modular Functions of One Variable, IV (Proc. Internat. Summer School, Univ. Antwerp, Antwerp, 1972), volume 476 of Lect. Notes Math., pages 53-73. Springer, Berlin, 1975.

[6] P. Deligne and M. Rapoport. Les schémas de modules de courbes elliptiques. In Modular Functions of One Variable, II (Proc. Internat. Summer School, Univ. Antwerp, Antwerp, 1972), volume 349 of Lect. Notes Math., pages 143-316. Springer, Berlin, 1973.

[7] C.L. Douglas, J. Francis, A.G. Henriques, and M.A. Hill, editors. Topological Modular Forms, volume 201 of Math. Surv. Monogr. Amer. Math. Soc., Providence, RI, 2014.

[8] D. Eisenbud. Commutative Algebra, volume 150 of Grad. Texts Math. SpringerVerlag, New York, 1995. With a view toward algebraic geometry.

[9] P. Goerss. Quasi-coherent sheaves on the moduli stack of formal groups. Available at http://www.math.northwestern.edu/ pgoerss/papers/modfg.pdf.

[10] P.G. Goerss. Topological modular forms [after Hopkins, Miller and Lurie]. Astérisque, (332): Exp. No. 1005, viii, 221-255, 2010. Séminaire Bourbaki. Volume 2008/2009. Exposés 997-1011.

[11] A. Grothendieck. Éléments de géométrie algébrique. IV. Étude locale des schémas et des morphismes de schémas. I. Publ. Math., Inst. Hautes Étud. Sci., (20):259, 1964.

[12] M. Hill and T. Lawson. Topological modular forms with level structure. Invent. Math. 203(2):359-416, 2016.

[13] M. Hopkins. Complex oriented cohomology theories and the language of stacks. Course Notes. 1999.

[14] M.J. Hopkins and M. Mahowald. From elliptic curves to homotopy theory. In Topological Modular Forms, volume 201 of Math. Surv. Monogr., pages 261285. Amer. Math. Soc., Providence, RI, 2014.

[15] M.J. Hopkins. Minimal atlases of real projective spaces. In Algebraic Topology (Arcata, CA, 1986), volume 1370 of Lect. Notes Math., pages 171-192. Springer, Berlin, 1989.

[16] M.J. Hopkins and H.R. Miller. Elliptic curves and stable homotopy I. In Topological Modular Forms, volume 201 of Math. Surv. Monogr., pages 209260. Amer. Math. Soc., Providence, RI, 2014. 
[17] J. Konter. The homotopy groups of the spectrum tmf. arXiv:1212.3656, 2012.

[18] T. Lawson and N. Naumann. Commutativity conditions for truncated BrownPeterson spectra of height 2. J. Topol., 5(1):137-168, 2012.

[19] T. Lawson and N. Naumann. Strictly commutative realizations of diagrams over the Steenrod algebra and topological modular forms at the prime 2. Int. Math. Res. Not., (10):2773-2813, 2014.

[20] J. Lurie. Chromatic homotopy theory, 2010. Course notes available at http://math.harvard.edu/ lurie/252x.html.

[21] M. Mahowald and C. Rezk. Topological modular forms of level 3. Pure Appl. Math. Q., 5(2, Special Issue: In honor of Friedrich Hirzebruch. Part 1):853-872, 2009.

[22] A. Mathew and L. Meier. Affineness and chromatic homotopy theory. J. Topol., 8(2):476-528, 2015.

[23] J.P. May and K. Ponto. More Concise Algebraic Topology. Chic. Lect. Math. University of Chicago Press, Chicago, IL, 2012. Localization, completion, and model categories.

[24] H. Miller. Sheaves, gradings, and the exact functor theorem. Available at http://www-math.mit.edu/ hrm/papers/bcat.pdf.

[25] J.W. Milnor and J.C. Moore. On the structure of Hopf algebras. Ann. Math. (2), 81:211-264, 1965.

[26] R.E. Mosher and M.C. Tangora. Cohomology Operations and Applications in Homotopy Theory. Harper \& Row, Publishers, New York, London, 1968.

[27] N. Naumann. The stack of formal groups in stable homotopy theory. Adv. Math., 215(2):569-600, 2007.

[28] D.C. Ravenel. Complex Cobordism and Stable Homotopy Groups of Spheres, volume 121 of Pure Appl. Math. Academic Press Inc., Orlando, FL, 1986.

[29] C. Rezk. Supplementary notes for math 512. 2007. Available at http://www. math.uiuc.edu/ rezk/512-spr2001-notes.pdf.

[30] J.H. Silverman. The Arithmetic of Elliptic Curves, volume 106 of Grad. Texts Math. Springer, Dordrecht, second edition, 2009.

[31] V. Stojanoska. Duality for topological modular forms. Doc. Math. J. DMV, (17):271-311, 2012.

Akhil Mathew amathew@math.harvard.edu

Department of Mathematics, Harvard University, Cambridge, MA, USA 